\title{
A Practical Guide to the Treatment of Dravet Syndrome with Anti-Seizure Medication
}

\author{
Adam Strzelczyk $^{1,2}$ - Susanne Schubert-Bast ${ }^{1,2,3} \mathbb{D}$
}

Accepted: 9 January 2022 / Published online: 14 February 2022

(c) The Author(s) 2022

\begin{abstract}
Dravet syndrome is a severe developmental and epileptic encephalopathy characterised by refractory seizures and cognitive dysfunction. The treatment is challenging, not least because the seizures are highly drug resistant, requiring multiple anti-seizure medications (ASMs), while some ASMs can exacerbate seizures. Initial treatments include the broad-spectrum ASMs valproate (VPA), and clobazam (CLB) in some regions; however, they are generally insufficient to control seizures. With this in mind, three adjunct ASMs have been approved specifically for the treatment of seizures in patients with Dravet syndrome: stiripentol (STP) in 2007 in the European Union and 2018 in the USA, cannabidiol (CBD) in 2018/2019 (in combination with CLB in the European Union) and fenfluramine (FFA) in 2020. These "add-on" therapies (mostly to VPA/ CLB) are used as escalation therapies, with the choice dependent on availability in different countries, patient characteristics and caregiver preferences. Topiramate is also frequently used, with evidence of efficacy in Dravet syndrome, and there is anecdotal evidence of efficacy with bromide, which is frequently used in Germany and Japan. With a growing treatment landscape for Dravet syndrome, there can be practical challenges for clinicians, particularly with issues associated with polypharmacy. This practical guide provides an overview of these main ASMs including their indications/contraindications, mechanism of action, efficacy, safety and tolerability profile, dosage requirements, and laboratory and clinical parameters to be evaluated. Standard laboratory and clinical parameters include blood counts, liver function tests, serum concentrations of ASMs, monitoring the growth of children, as well as weight loss and acceleration of behavioural problems. Regular cardiac monitoring is also important with FFA as it has previously been associated with cases of cardiac valve disease when used in adults at high doses (up to $120 \mathrm{mg}$ /day) in combination with phentermine as a therapy for obesity. Importantly, no signs of heart valve disease have been documented to date at the low doses used in patients with developmental and epileptic encephalopathies. In addition, potential drug-drug interactions and their consequences are a key consideration in everyday practice. Interactions that potentially require dosage adjustments to alleviate adverse events include the following: STP + CLB resulting in increased plasma concentrations of CLB and its active metabolite norclobazam may increase somnolence, and an interaction with STP and VPA may increase gastrointestinal adverse events. Cannabidiol has a bi-directional interaction with CLB producing an increase in plasma concentrations of 7-OH-CBD and norclobazam resulting in the potential for increased somnolence and sedation. In addition, CBD is associated with elevations of liver transaminases particularly in patients taking concomitant VPA. The interaction between FFA and STP requires a dose reduction of FFA. Furthermore,
\end{abstract}

Adam Strzelczyk

strzelczyk@med.uni-frankfurt.de

1 Epilepsy Center Frankfurt Rhine-Main, Center of Neurology and Neurosurgery, Goethe-University Frankfurt, Schleusenweg 2-16 (Haus 95), 60528 Frankfurt am Main, Germany

2 LOEWE Center for Personalized and Translational Epilepsy Research (CePTER), Goethe-University Frankfurt, Frankfurt am Main, Germany

3 Department of Neuropediatrics, Goethe-University Frankfurt, Frankfurt am Main, Germany 
concomitant administration of VPA with topiramate has been associated with encephalopathy and/or hyperammonaemia. Finally, we briefly describe other ASMs used in Dravet syndrome, and current key clinical trials.

\section{Graphical abstract}

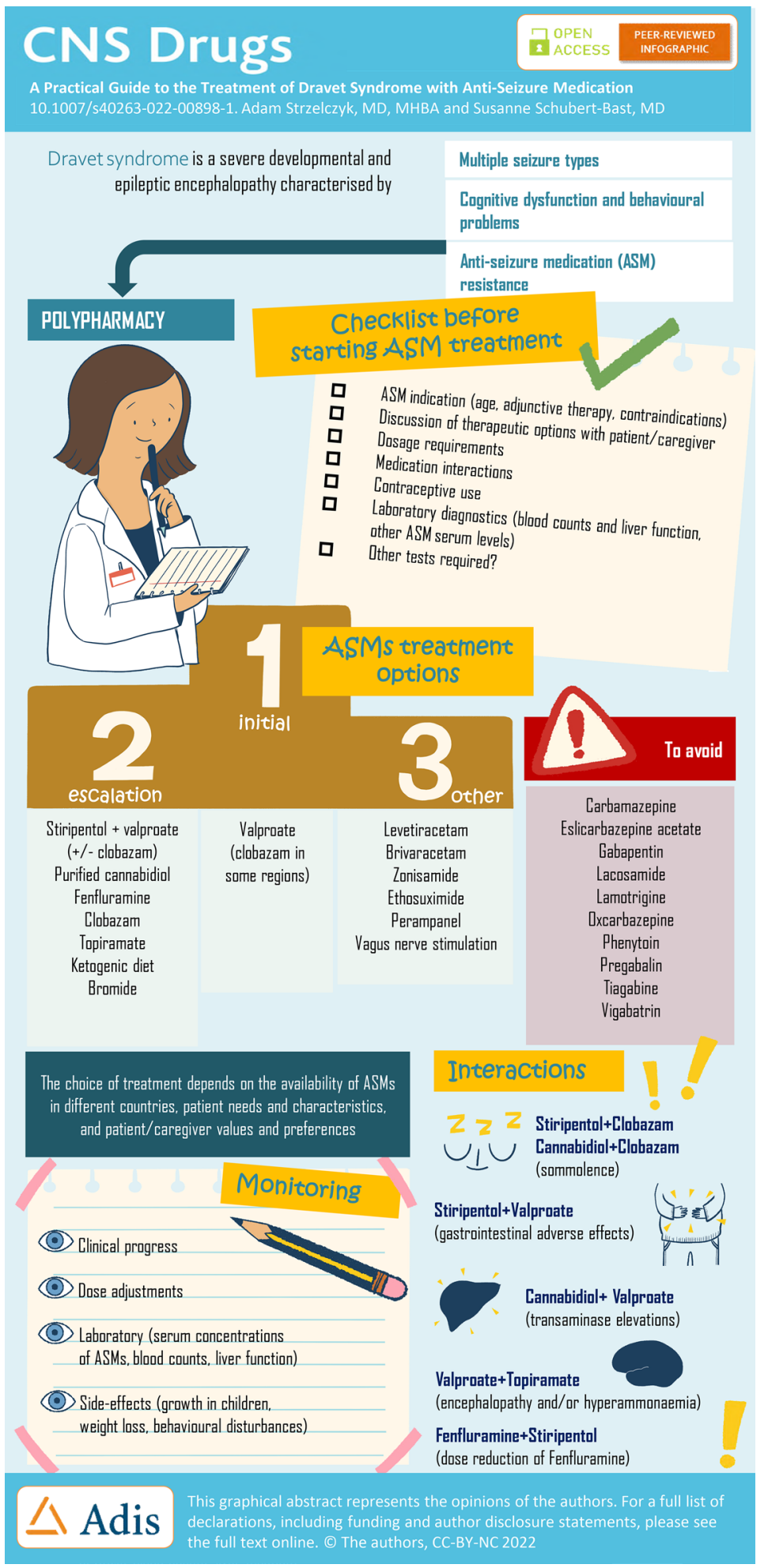




\section{Key Points}

Patients with Dravet syndrome are treated with multiple anti-seizure medications to control seizures including valproate (VPA), and clobazam (CLB in some regions), as initial therapy, and stiripentol (STP), cannabidiol (CBD) or fenfluramine (FFA) as escalation therapies; alternatives include topiramate (TPM), bromide, levetiracetam, brivaracetam, zonisamide, ethosuximide and perampanel.

Because polypharmacy is the norm in Dravet syndrome, there are practical pitfalls that include changes in serum concentrations of anti-seizure medications and the potential for increased adverse events, requiring careful monitoring and possible dose adjustments.

Key interactions that may require dosage adjustments to alleviate adverse events include STP+CLB (somnolence), STP+VPA (gastrointestinal adverse events), CBD+CLB (somnolence), CBD+VPA (liver transaminase elevations), FFA+STP (dose reduction of FFA) and VPA+TPM (encephalopathy and/or hyperammonaemia).

\section{Introduction}

Dravet syndrome (DS) is a severe developmental and epileptic encephalopathy characterised by drug-resistant seizures and cognitive dysfunction, negatively impacting on the quality of life of the patients and their families [1,2]. In the majority of cases, it is caused by heterozygous loss-of-function variants in the $S C N 1 A$ gene, resulting in substantially decreased levels of the corresponding functional protein, the $\alpha-1$ subunit of the neuronal, voltage-gated sodium channel $\mathrm{Na}_{\mathrm{V}} 1.1$ [3]. $\mathrm{Na}_{\mathrm{V}} 1.1$ is a key sodium channel in the central nervous system that is highly expressed in many GABAergic inhibitory neurons, and the impairment of the production of this protein leads to hyperexcitability of the neuronal network [4].

As with other developmental and epileptic encephalopathies, the management of DS is challenging (Fig. 1). First, DS is associated with multiple seizure types that evolve over time [2, 3,5]. Seizures typically begin before the age of 1 year, in a formerly healthy infant, usually with tonic-clonic or clonic febrile seizures. Between the ages of 1 and 5 years, afebrile seizure types emerge including generalised motor, atypical, myoclonic and absence. Seizures are often prolonged, and status epilepticus (SE) is common especially in the patient's first 5 years. Beyond the age of 5 years, seizure frequency begins to decrease, with a gradual transition with age to nocturnal seizures, which are the main type of seizures in adults. Nocturnal seizures can also pose challenges including sleep disturbances for both patients and their family members [6]. Nocturnal seizures, SE and sudden unexpected death in epilepsy result in a higher mortality rate compared with patients with other epilepsy [7]. In addition to seizures, patients experience varying degrees of cognitive dysfunction and behavioural problems. As such, even though the seizure burden may dissipate with age, there remains a high level of dependency throughout the patient's life [6].

Another challenge is that some anti-seizure medications (ASMs) can exacerbate seizures in DS (Fig. 2), and therefore an early diagnosis is important to make sure patients are treated appropriately (Fig. 1). In addition, high seizure frequency is associated with a lower quality of life, and may be correlated with cognitive dysfunction, further highlighting the importance of a prompt diagnosis and timely treatment of seizures [8-12].

Patients are usually treated with multiple ASMs in an attempt to control seizures, with valproate (VPA) and clobazam (CLB) generally being inadequate (Fig. 2) [13-15]. With this in mind, three ASMs specifically approved as adjunct therapies in patients with DS have become available in the last decade: stiripentol (STP), which was approved in 2007 in the European Union (EU) and 2018 in the USA, cannabidiol (CBD) approved in 2018/2019 and fenfluramine (FFA) approved in 2020 (Fig. 2) [13, 14, 16, 17]. These treatments are "add-on" therapies (most commonly to VPA and CLB), with the choice dependent on availability and recommendations in different countries, among other factors (Fig. 2) [13, 14, 16, 17]. Overall, an individualised approach to treatment should be employed, taking into account patient needs, together with patient/caregiver values and preferences to collaboratively choose the most appropriate treatment $[18,19]$.

With a growing treatment landscape for DS, the complexity surrounding treatment decisions increases, causing practical challenges for clinicians (Fig. 1). Herein, we provide a practical guide to the main ASMs that have evidence of efficacy in the treatment of seizures in DS. We provide an overview of the indications and contraindications, their mechanism of action, efficacy, safety and tolerability profile, dosage requirements, and laboratory and clinical parameters to be evaluated. In addition, because of the need for polypharmacy, potential drug-drug interactions (in particular with other relevant ASMs and with hormonal contraceptives) and their consequences are described. Figure 3 provides a checklist of these considerations, with signposts to the relevant tables that provide further details, in addition to the narrative below. The focus of this guide is on the pharmacological treatment of seizures with ASMs, although it should be noted that patients with DS may also use other medications 
Fig. 1 Treatment challenges in patients with Dravet syndrome (DS). ASMs anti-seizure medications

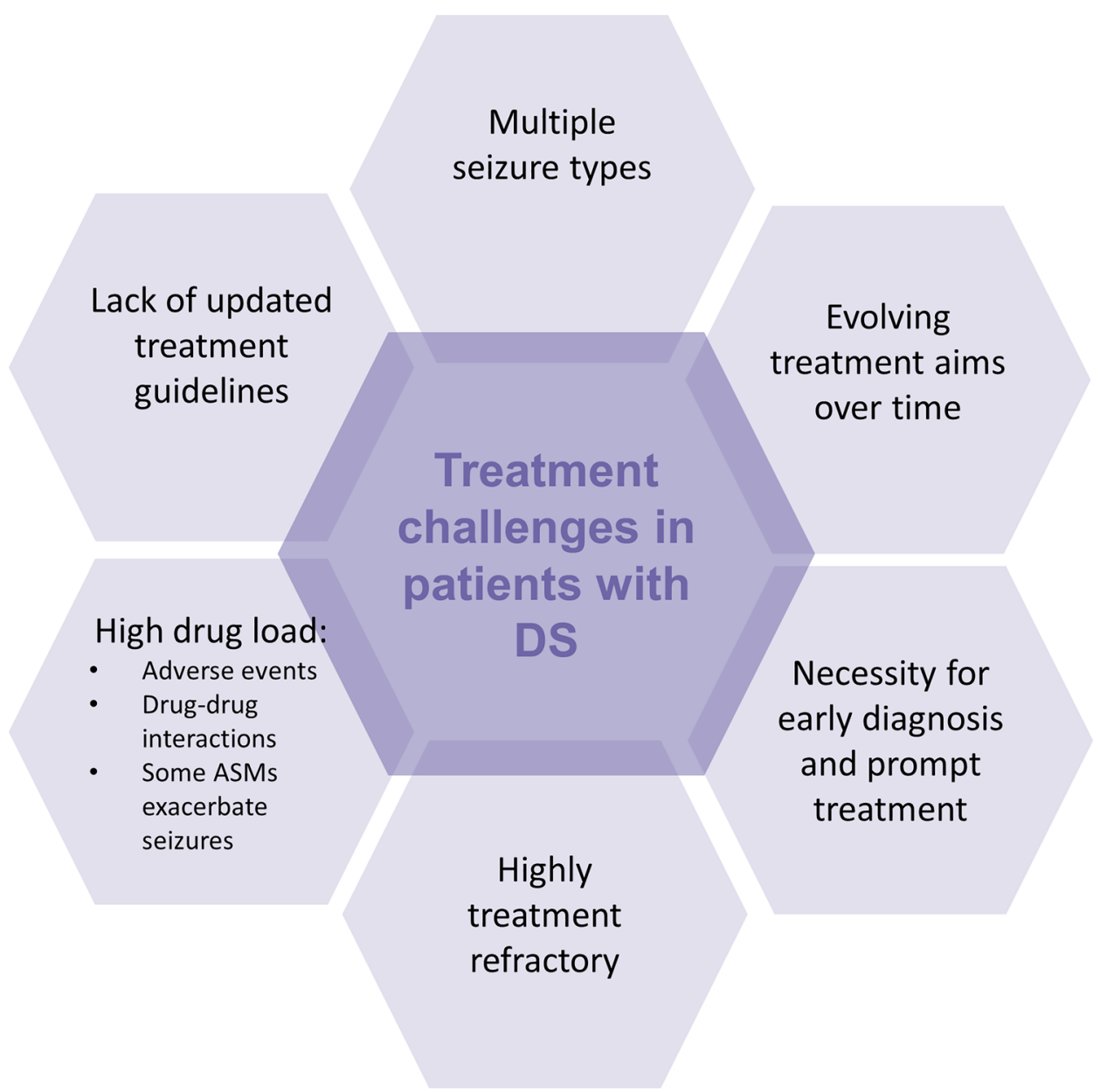

for behavioural and sleep problems, as well as emergency rescue medication [20, 21]. In addition, non-pharmacological interventions, including the ketogenic diet and vagus nerve stimulation in carefully selected patients, can also help alleviate seizures but are not covered in this guide.

\section{Valproate (VPA)}

Valproate, a widely used agent for epilepsy in all ages, is the first-line treatment for patients with DS (Fig. 2). Valproate is a broad-spectrum ASM effective across a range of focal and generalised seizure types including tonic-clonic, myoclonic, tonic and absence seizures (see Table 1 for the indications in the EU/UK and the USA). The wide-ranging activity is likely a reflection of its complex mechanism of action modulating various targets implicated in the development of seizures, including the inhibitory neurotransmitter GABA, voltage-gated sodium channels and histone deacetylase (Fig. 4) [22]. Valproate has proved effective in generalised and unclassifiable epilepsies [23, 24], and is generally well tolerated, an important contributing factor behind its extensive use in epilepsy.

Valproate is available in a variety of formulations including tablets (regular, crushable, gastro-resistant and prolonged release) and granules (modified release), oral solution (liquid and syrup), as well as a solution for injection or infusion. The starting dose of VPA is typically between 10 and $15 \mathrm{mg} / \mathrm{kg} / \mathrm{day}$, divided into two doses for the generally favoured prolonged-release formulation or three doses otherwise, followed by gradual increases to target doses in the range of 25 to a maximum of $60 \mathrm{mg} / \mathrm{kg} /$ day according to clinical response and tolerability. In cases where acceptable clinical response has not been attained, plasma concentrations of VPA should be checked to see if they are in the generally accepted therapeutic range of $50-100 \mathrm{mcg} / \mathrm{mL}$. Of note, VPA is also being trialled in patients with drugresistant epilepsy for long-term intra-cerebroventricular drug delivery, a novel method using an implantable infusion system that administers the drug into the cerebrospinal fluid [25].

Valproate has a generally acceptable safety profile. The most common adverse events (AEs) include gastrointestinal 


Initial therapy
Valproate
Clobazam (in
some regions);
add the other if
first choice
ineffective

Escalation therapy
Based on strong RCT evidence
- Stiripentol (+/- valproate / clobazam)
- Purified cannabidiol
- Fenfluramine
Alternatives (weaker evidence)
- Clobazam
- Topiramate
- Ketogenic diet
- Bromide

\section{Other available therapies}

Levetiracetam, brivaracetam, zonisamide, ethosuximide, perampanel OR consider VNS

\begin{tabular}{|lr|}
\hline \multicolumn{2}{|c|}{ ASMs to avoid } \\
\hline Carbamazepine & Oxcarbazepine \\
Eslicarbazepine acetate & Phenytoin \\
Gabapentin & Pregabalin \\
Lacosamide & Tiagabine \\
Lamotrigine & Vigabatrin \\
\hline
\end{tabular}

Fig. 2 Treatment pathway in patients with Dravet syndrome. Adapted from Cross et al. [124]. The choice of treatment is dependent on the drug availability and recommendations in different countries, patient

disturbances (nausea, vomiting and abdominal pain and diarrhoea), headache, somnolence, tremor and asthenia, although these are generally mild to moderate in severity and reduce over time [26, 27]. However, VPA has been associated, albeit rarely, with serious and fatal hepatotoxicity, with children $<2$ years of age (especially those taking multiple ASMs) and people with mitochondrial disorders caused by mutations of the mitochondrial DNA Polymerase $\gamma$ (POLG) gene at particular risk [26, 27]. As such, VPA is contraindicated in patients with hepatic disease or significant hepatic dysfunction and those with hereditary POLGl-associated mitochondrial disease; of note, rare cases of POLG1 mutations in patients with DS have been reported [28, 29]. Before initiating VPA, liver function tests are required to exclude liver disease and patients should be monitored regularly thereafter, particularly during the first 6 months [26, 27]. A physical examination and a medical history should also be conducted as liver function tests are not always abnormal, and instead, non-specific symptoms including malaise, weakness, lethargy, facial oedema, anorexia and vomiting, as well as a loss of seizure control, have been reported to occur prior to cases of serious/fatal hepatotoxicity [26, 27]. Valproate is also associated with coagulation disorders (including thrombocytopenia, decreases in von Willebrand's factor and platelet dysfunction) and hyperammonaemia. Therefore, in addition to liver enzymes, laboratory tests should include blood count and ammonia; tests should be performed before treatment initiation and then repeated after 6 weeks, 3 months and 6 months after treatment initiation, and then in yearly intervals (Table 2). In addition, coagulation parameters, platelet count and platelet function should be tested if surgery is planned.

There is a paucity of studies evaluating VPA as monotherapy in patients with DS, with three retrospective studies reporting $>50 \%$ responder rates of $23-52 \%$ [30-32]. needs and characteristics, and patient/caregiver values and preferences. ASMs anti-seizure medications, RCT randomised controlled trial, $V N S$ vagus nerve stimulation

However, VPA alone is generally insufficient to control seizures in DS and persisting seizures leads to further add-on treatments. Therefore, as polypharmacy is the norm in DS, an awareness of the consequences of potential drug-drug interactions and the potential for AEs with other ASMs is a key consideration (Table 3). Of note, concomitant administration with topiramate (TPM) has been associated with encephalopathy and/or hyperammonaemia; the signs and symptoms should be carefully monitored especially in patients with pre-existing encephalopathy (Table 3) [26, 27]. Valproate is an inhibitor of a broad range of hepatic enzymes (cytochrome P450s, uridine glucuronyl transferases and epoxide hydrolase, among others) that are involved in the metabolism of other ASMs, resulting in increased serum concentrations of these substrates including lamotrigine, phenobarbital, the metabolite 10,11-epoxide of carbamazepine, ethosuximide and rufinamide. While these ASMs are rarely used in DS, it may still be worth noting that increased monitoring of ASM concentrations and dosage adjustments are required when these drugs are initiated or withdrawn. In particular, an increased risk of serious skin conditions (Stevens-Johnson syndrome and toxic epidermal necrolysis) has been reported with concomitant lamotrigine, requiring a slow titration of lamotrigine at initiation. Furthermore, the use of oestrogen-containing contraceptives can lead to decreased efficacy of VPA requiring monitoring (Table S1 of the Electronic Supplementary Material [ESM]).

\section{Clobazam (CLB)}

As with VPA, CLB is widely used for the treatment of developmental and epileptic encephalopathies including DS, owing to its favourable tolerability, safety and broadspectrum anti-seizure activity. In the EU, CLB has a broad 


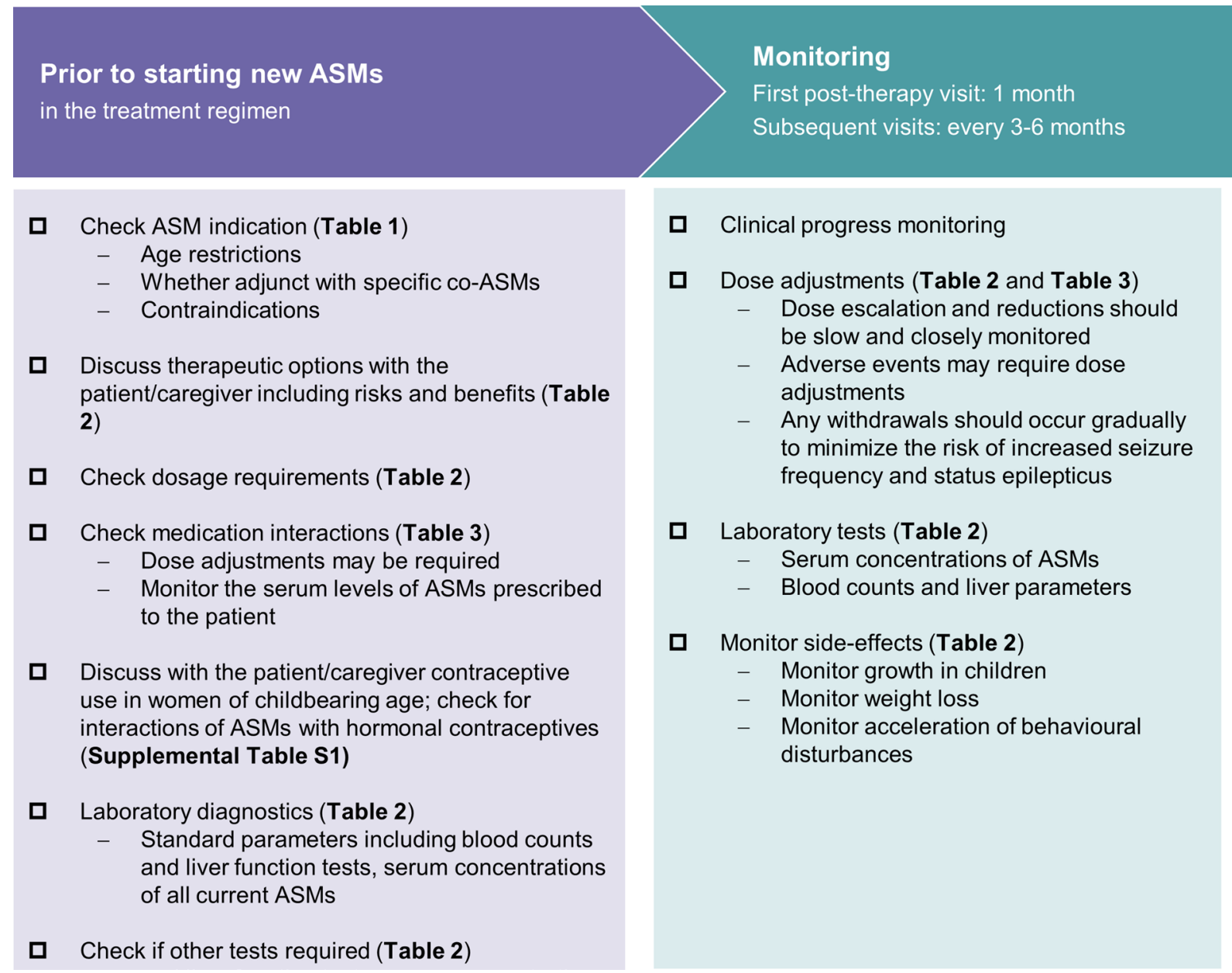

Fig. 3 Checklist to consider through Dravet syndrome treatment. ASMs anti-seizure medications

indication as an adjunct treatment for epilepsy [33], while in the USA it is generally used off-label, being only relatively recently approved as an adjunctive treatment of seizures associated with Lennox-Gastaut syndrome (LGS) (Table 1) [34]. Clobazam is a 1,5-benzodiazepine, and as with other benzodiazepines its effects are mediated by binding to the GABA-A receptor, among other mechanisms (Fig. 4). However, CLB has a structure that is different from 1,4-benzodiazepines, with a greater selectivity for $\alpha 2$-GABA-A than $\alpha 1-G A B A-A$ subunits, which results in fewer sedative effects and a lower propensity for efficacy tolerance (i.e. losing efficacy over time) [35].

Clobazam is administered orally, available in the EU and USA as oral tablets and an oral suspension, while an oral soluble film was approved in the USA in 2018 [36]. The initial dose of CLB is $0.2 \mathrm{mg} / \mathrm{kg} / \mathrm{day}$ divided into twice daily, followed by gradual increases to reach target doses of $0.3-1 \mathrm{mg} / \mathrm{kg} / \mathrm{day}$, up to a maximum of $2 \mathrm{mg} / \mathrm{kg} /$ day. Of note, much of the anti-seizure activity of CLB may be mediated through its active metabolite, norclobazam
( $N$-desmethyl-clobazam [nCLB]). Because serum concentrations of CLB and nCLB only reach steady state after 5 and 9 days, respectively, dose escalation should not occur more than weekly $[33,34]$. There is a high level of intra-individual variability in the plasma concentrations of CLB and nCLB, but monitoring is advisable with regard to specific drug-drug interactions. $\mathrm{N}$-desmethyl-clobazam is primarily metabolised by CYP2C19, and patients who are CYP2C19 poor metabolisers (i.e. individuals with variants in $C Y P 2 C 19$ that lead to reduced or no activity) are recommended to have half the dosage of CLB because of an increased risk of adverse reactions [34].

There is gold-standard evidence from a randomised controlled trial (RCT) related to the efficacy of CLB for LGS [37], and also in conjunction with STP, CBD and FFA in DS (see below), but otherwise the published evidence is limited to two retrospective studies that reported $>50 \%$ responder rates of $27.6 \%$ and $45.5 \%$ in patients with DS treated with adjunct CLB [30, 31]. Clobazam has a fairly benign safety profile: AEs reported in the phase III trial in patients with 


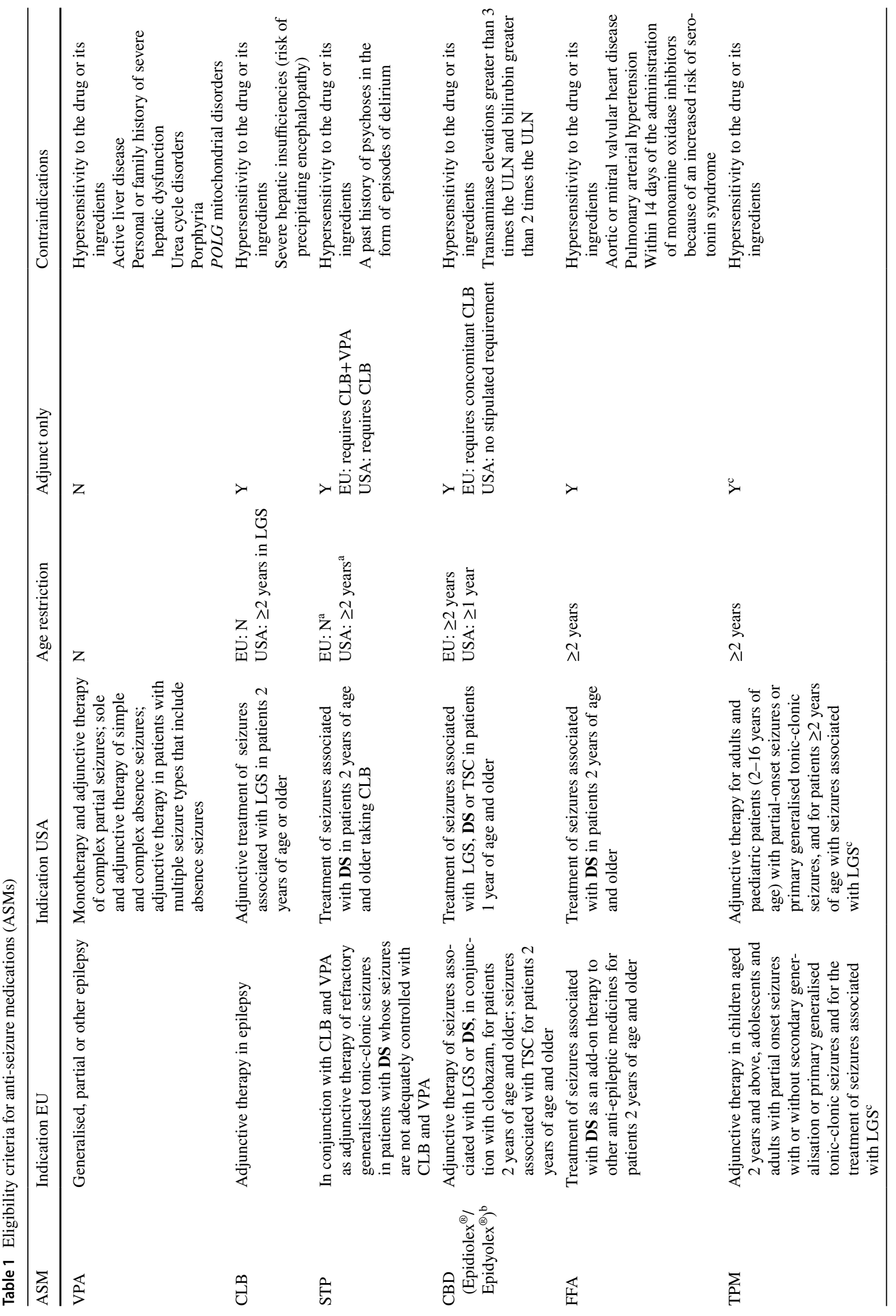




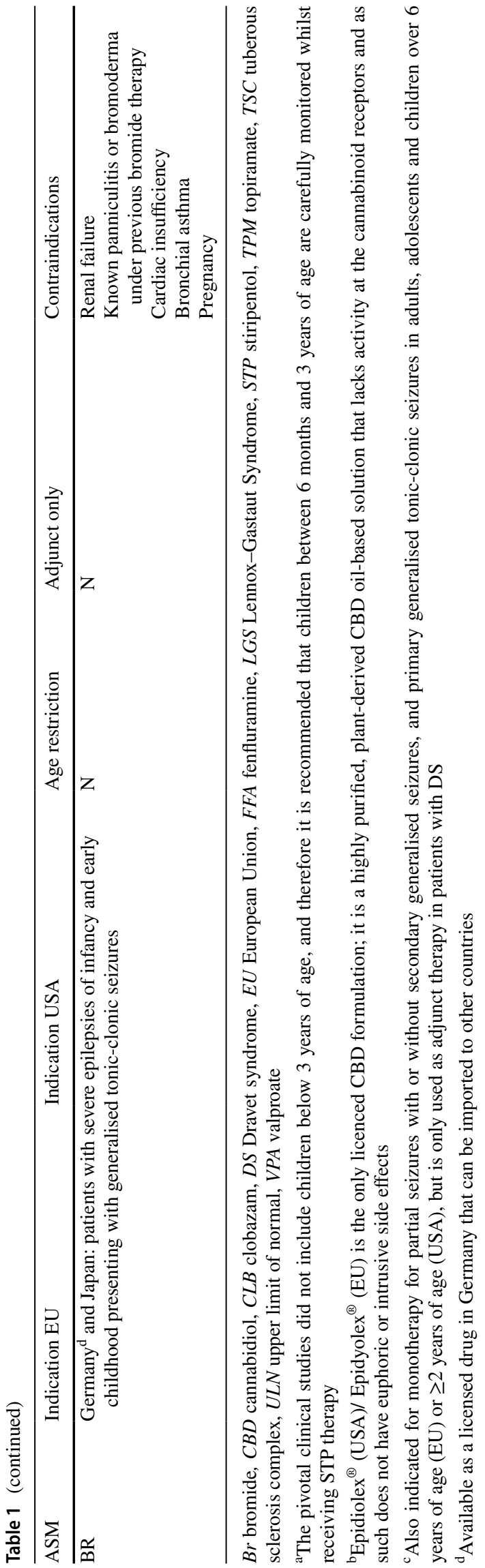

LGS were consistent with other experiences, and included somnolence, pyrexia, upper respiratory infections, lethargy, drooling and constipation [37, 38]. Clobazam can also be associated with respiratory depression, especially at high doses or with concomitant opioids. No laboratory tests above those recommended for VPA are required (Table 2). Addition of CLB to VPA may increase the plasma concentrations of VPA, and clinical monitoring is recommended with a view to a possible dose adjustment of CLB (Table 3) [33, 34]. Interactions with other relevant ASMs are discussed in the corresponding sections below. Additionally, it is noteworthy that lower doses of drugs metabolised by CYP2D6 (e.g. dextromethorphan, pimozide, paroxetine, nebivolol) may be required when used with CLB and lower doses of CLB may be required with strong or moderate CYP2C19 inhibitors (e.g. fluconazole, fluvoxamine, ticlopidine, omeprazole) $[33,34]$.

\section{Stiripentol (STP)}

Stiripentol, approved in 2007 in the EU and 2018 in the USA, was the first therapy indicated specifically for the treatment of seizures in patients with DS in conjunction with CLB (with VPA + CLB in the EU). As with other ASMs, STP likely has multiple mechanisms of action connected to its anti-seizure properties that are not fully elucidated [39]. One such mechanism is potentiating GABAergic transmission, acting as a positive allosteric modulator of GABA-A receptors at a site that is different from benzodiazepines; therefore, the combination of STP with a benzodiazepine such as CLB, both acting at different sites, leads to a stronger additive effect compared with either drug alone [40]. In addition, STP increases plasma concentrations of CLB and nCLB via inhibition of CYP enzymes, and these enhanced concentrations may also indirectly contribute to the anti-seizure activity (see below) [41, 42].

Stiripentol is available as hard capsules or a powder for oral suspension. The dose of STP should be escalated gradually, starting with $20 \mathrm{mg} / \mathrm{kg} / \mathrm{day}$ for 1 week, then $30 \mathrm{mg} /$ $\mathrm{kg} /$ day for 1 week, up to a maximum of $50 \mathrm{mg} / \mathrm{kg} /$ day in children titrated over an extra 1-4 weeks depending on age (Table 2). Stiripentol has a complex pharmacokinetic profile, requiring consideration of not just weight and drug-drug interactions, but also of age: in older children, adolescents and adults, the dosage is generally lower, in the range of $20-25 \mathrm{mg} / \mathrm{kg} / \mathrm{day}$, due to the lower rate of metabolism of adults compared with younger children [43]. The dosage is administered two or three times daily, and because it degrades rapidly in an acidic environment it should be taken with food in order to limit the exposure to gastric acid in an empty stomach $[44,45]$. In addition, STP should not be taken with dairy products, carbonated drinks or fruit juice 


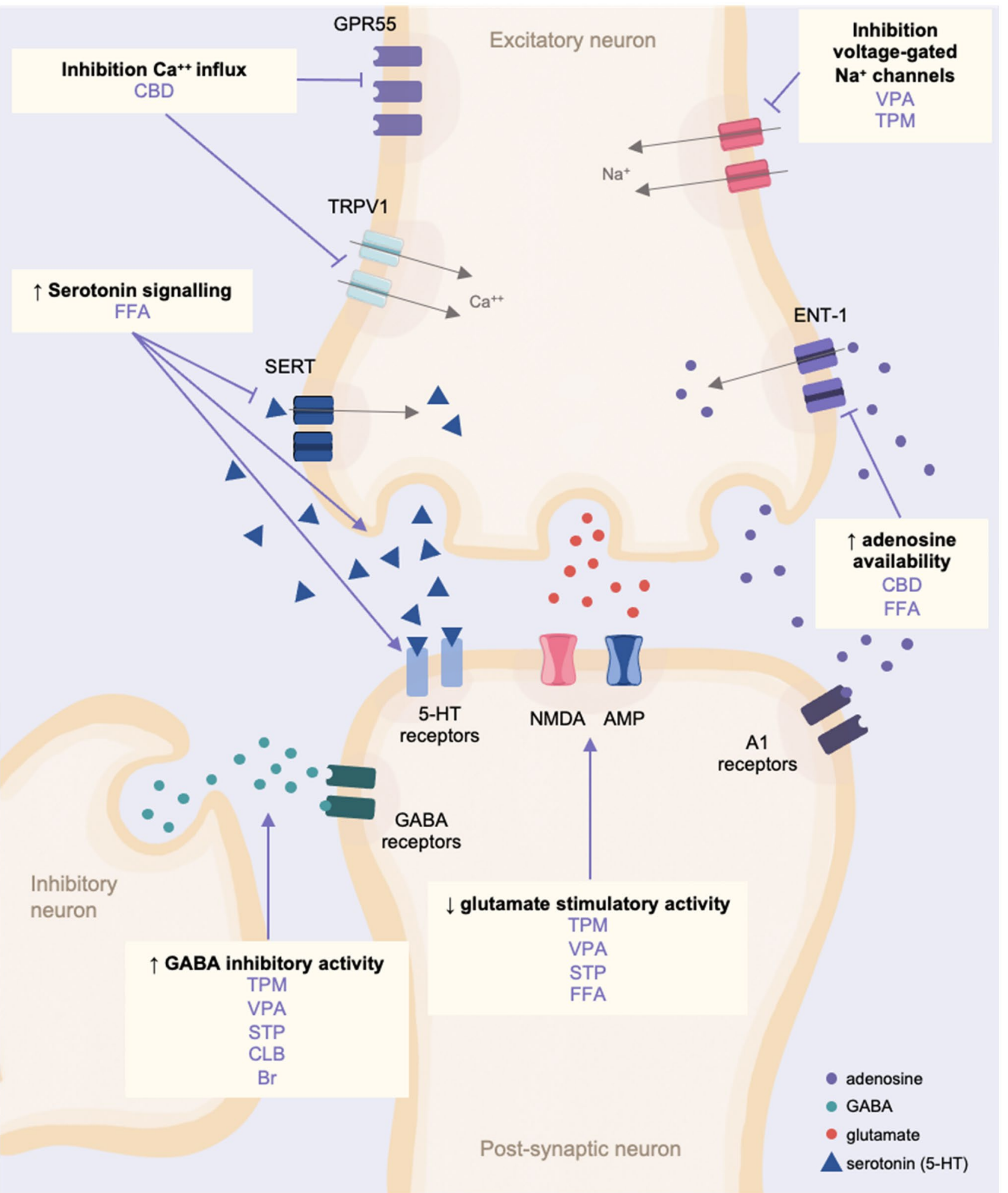

Fig. 4 Simplified schematic of the main known mechanisms of action of the anti-seizure medications used in Dravet syndrome. $B r$ bromide, $C a++$ calcium, $C B D$ cannabidiol, $C L B$ clobazam, ENT-1 equilibrative nucleoside transporter 1, FFA fenfluramine, GABA gamma-amin- obutyric acid, GRP55 G protein-coupled receptor 55, $\mathrm{Na}$ + sodium, $N M D A \mathrm{~N}$-methyl-D-aspartate, SERT serotonin transporter, STP stiripentol, TPM topiramate, TRPVI transient receptor potential vanilloid subtype $1, V P A$ valproate, $\uparrow$ increased, $\downarrow$ decreased 


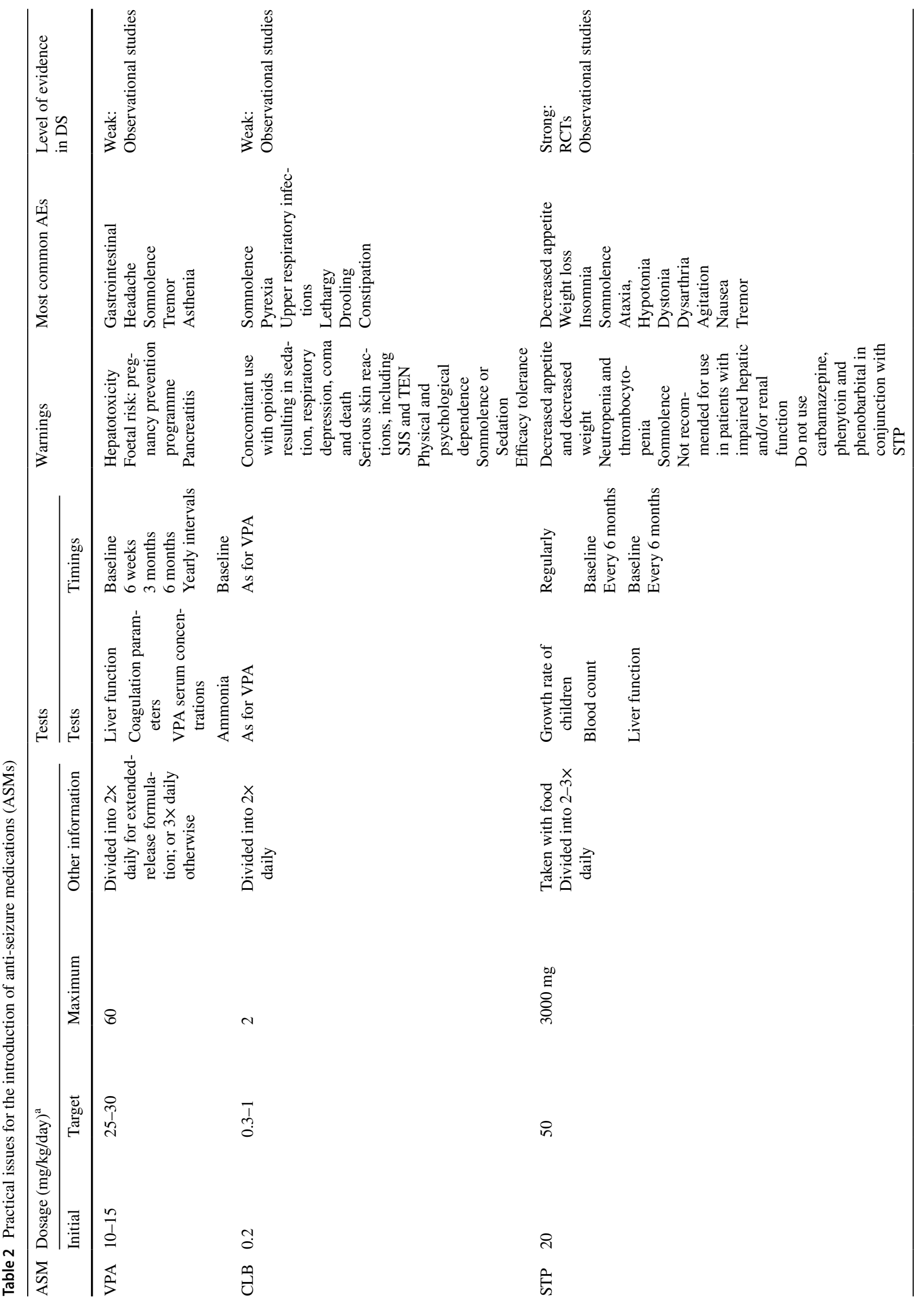




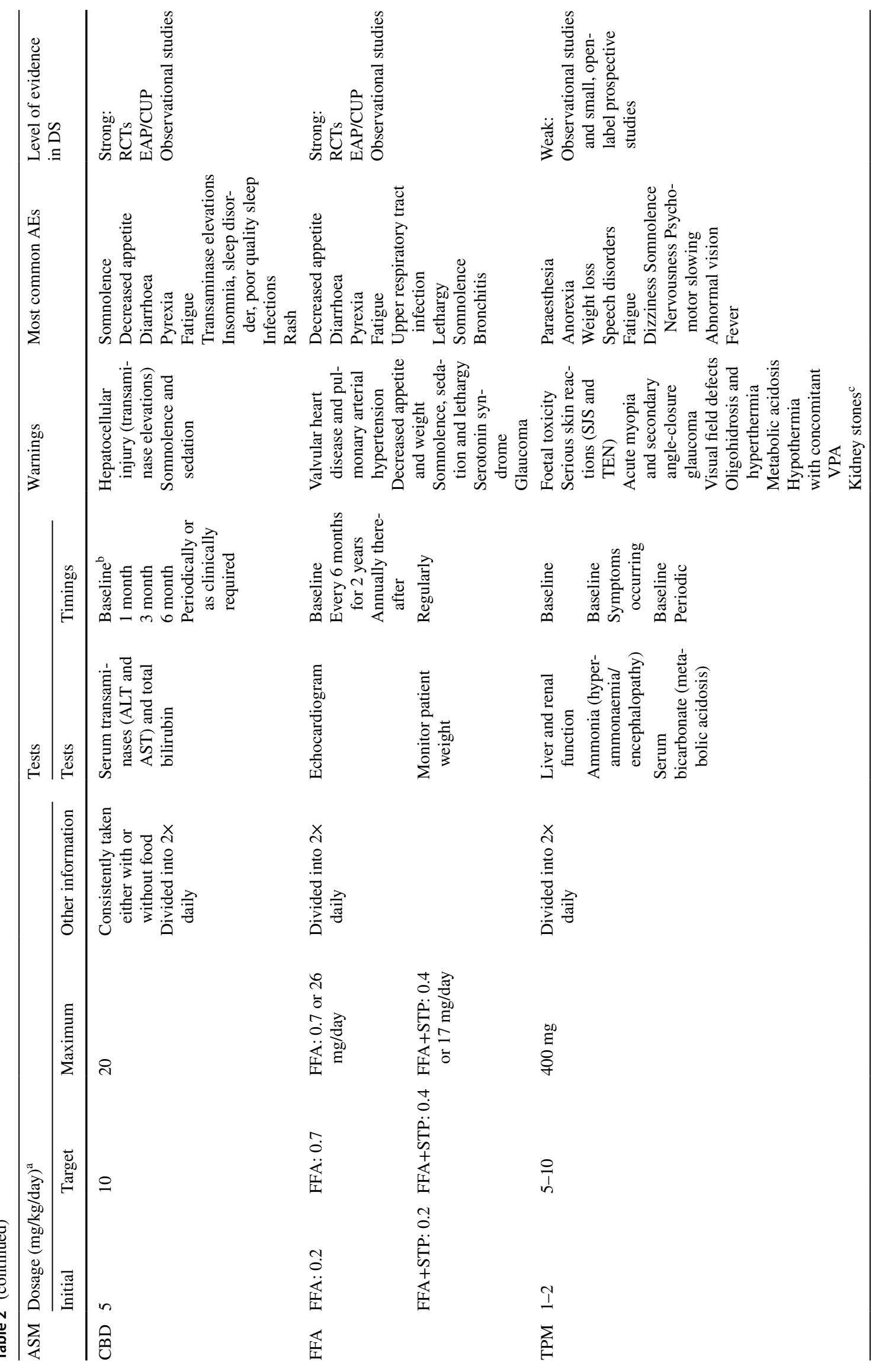




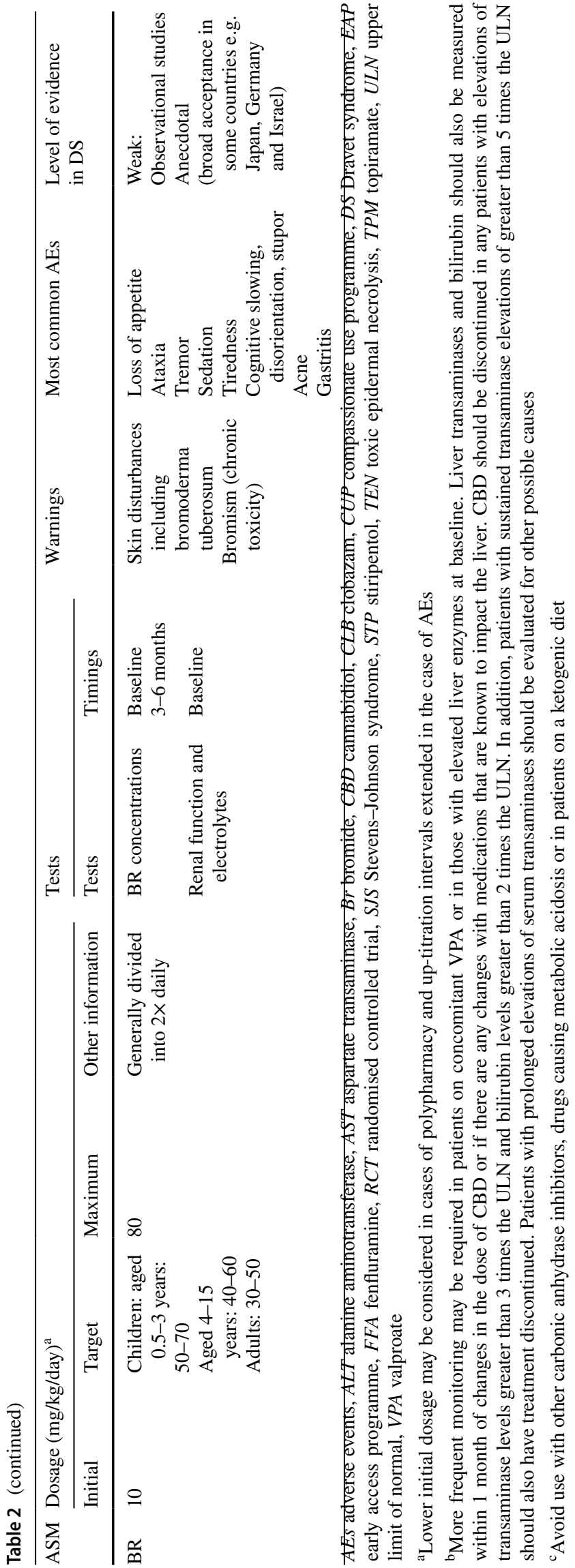

$[44,45]$. Furthermore, food and drinks that contain caffeine or theophylline should be avoided as STP may affect their clearance from the body [44, 45].

The efficacy and safety of STP as an adjunctive treatment with VPA and CLB have been demonstrated in two phase III, double-blind, placebo-controlled RCTs in patients with DS aged $\geq 3$ years: STP + VPA + CLB was associated with significantly higher $>50 \%$ responder rates compared with placebo+VPA+CLB $(71 \%$ vs $5 \%[p<0.0001]$ in STICLO-France $[46,47]$ and $67 \%$ vs $9 \%[p=0.0094]$ in STICLO-Italy) [48]. With a generally good tolerability and safety profile, the main AEs associated with the addition of STP in STICLO-France were drowsiness, hyperexcitability decreased appetite and weight decreased [47]. A further study from the French group in 46 children with DS demonstrated the long-term efficacy of STP + VPA + CLB over a median follow-up of 3 years; the number and duration of seizures significantly decreased, together with the number of convulsive SE events [49]. In addition, patients from this French cohort who initiated treatment with STP + VPA + CLB in childhood continued to see benefits into adulthood over a median duration of 18 years $(n=40$ patients followed); reductions in seizure (generalised tonicclonic) frequency and duration continued to be observed, with seizure-free periods of $1-5$ years seen in ten patients, while there were no SE events [50]. An open-label trial in Japan ( $n=24$ patients) [51,52] and a retrospective study in the USA ( $n=82$ children) ([42]) have also provided pivotal evidence of the long-term efficacy and safety of STP. Since then, a post-marketing surveillance study in Japan involving data from 410 patients with DS (aged $0.5-50$ years) reported responder rates of $43 \%$ for convulsive seizures, $55 \%$ for focal impaired awareness seizures, and $62 \%$ for generalised myoclonic seizures and/or generalised atypical absence seizures; the main AEs were somnolence (39\%) and a loss of appetite (25\%) [53]. A number of other observational studies have also confirmed the efficacy and safety of STP in realworld clinical practice across different age groups (including adults [54]) and with various follow-up periods, as recently reviewed $[1,39,55,56]$. However, studies also suggest that a significant proportion of patients may have inadequate seizure control over the long term, despite the use of this combination of ASMs [57, 58].

Because of the effects of STP on reducing appetite, it is important to monitor the weight of patients and the growth rate of children [44, 45]. In addition, as with other ASMs, STP is associated with neutropenia and thrombocytopenia and therefore complete blood counts should be obtained before initiating treatment and every 6 months thereafter; liver function tests are also recommended [44, 45]. A recent study has also highlighted the increased risk of hyperammonaemic encephalopathy in patients with DS who initiated treatment with STP in adulthood [59]. The increased 
Table 3 Drug-drug interactions for anti-seizure medication ${ }^{\mathrm{a}, \mathrm{b}, \mathrm{c}, \mathrm{d}}$

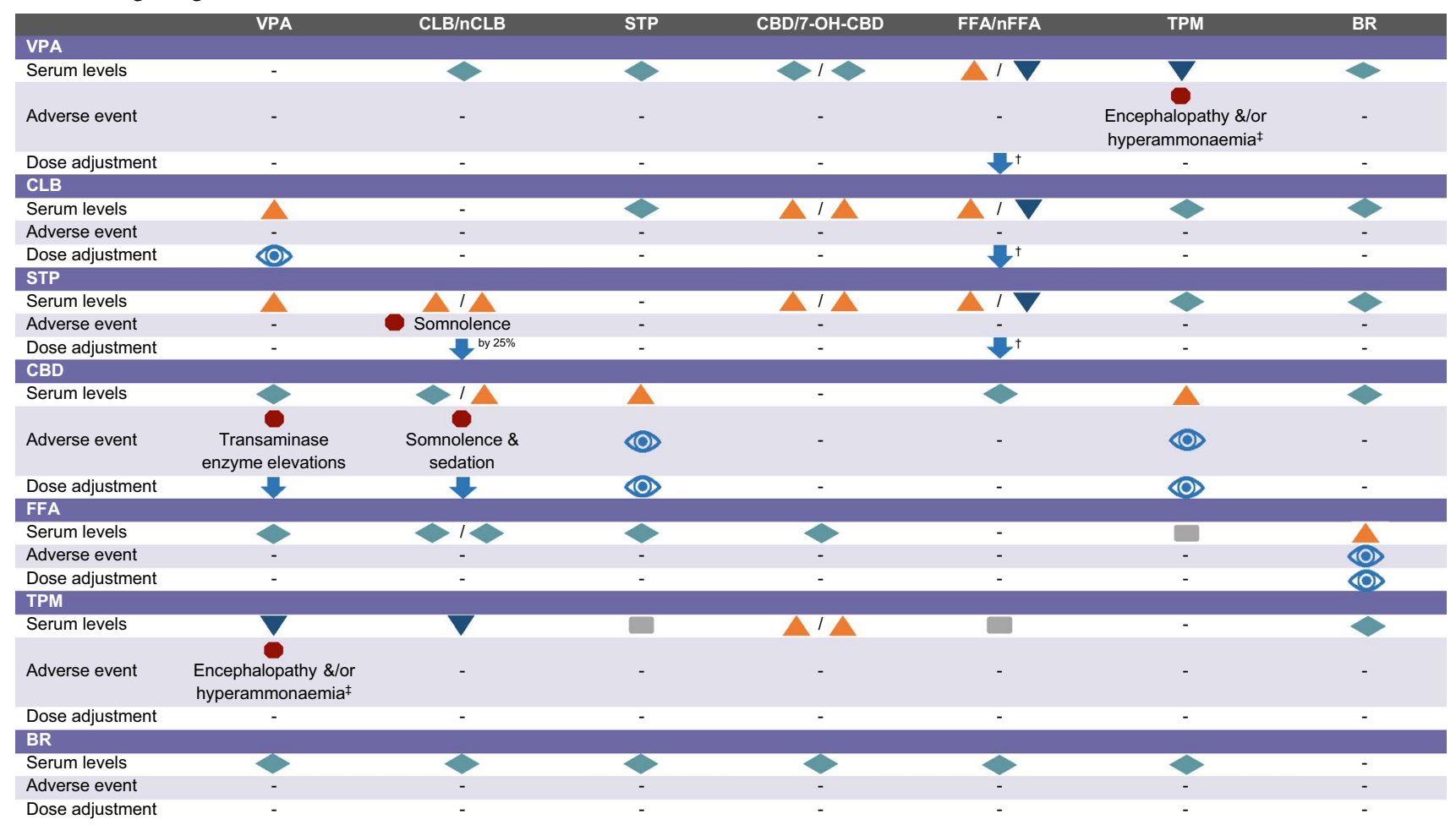

Adapted from Wheless et al. [21]

Serum concentrations: the interaction results in an increase of the ASM specified in the column heading; $\nabla$ decrease; $>$ no clinically relevant change; unknown/data not available (relates to the ASM specified in the column heading)

Dose adjustments: $\_$decrease in the dose of the ASM specified in the column heading may be required

Adverse events: increased risk of adverse events (relates to the ASM specified in the column heading)

(O) monitoring recommended for AEs and the need for dose adjustments of the ASM specified in the column heading due to potential changes in serum concentrations

7- $O H$ - $C B D$ 7-hydroxycannabidiol, $A S M$ anti-seizure medications, $B R$ bromide, $C B D$ cannabidiol, $C L B$ clobazam, $F F A$ fenfluramine, $n C L B$ $\mathrm{N}$-desmethyl-clobazam, $n F F A$ norfenfluramine, $S T P$ stiripentol, TPM topiramate, $V P A$ valproate

${ }^{a}$ Because of multiple interactions, it is advisable to monitor the serum concentrations of ASMs prescribed to the patient

${ }^{b}$ FFA dose is restricted to $0.4 \mathrm{mg} / \mathrm{kg} /$ day or $17 \mathrm{mg} /$ day when FFA is in combination with STP

${ }^{c}$ Expert opinion from Germany regarding the use of FFA+BR: BR concentrations increased in a proportion of patients after initiation of FFA. Dose reductions of bromide were made in most patients

${ }^{\mathrm{d}}$ Encephalopathy or hyperammonaemia in those on VPA/TPM requires discontinuation of either TPM or VPA

ammonia levels occurred despite dose reductions of VPA and CLB, but they were generally normalised with carnitine treatment [59].

Stiripentol increases the plasma concentration of VPA and CLB its active metabolite nCLB. In particular, dose reductions of CLB (of approximately $25 \%$ per week) may be required, especially in the event of AEs such as drowsiness, hypotonia and irritability in young children $[44,45]$. The interaction with VPA is more moderate, and a dose adjustment of VPA may not be needed, although an increase in gastrointestinal AEs including loss of appetite and loss of weight has been reported, with a suggested dose reduction of VPA of $30 \%$ every week $[44,45]$. The effects on VPA and
CLB are the result of STP being an inhibitor of cytochrome P450, which also has implications for other ASMs and other drugs $[44,45]$. Drugs to be generally avoided include immunosuppressants (e.g. tacrolimus, ciclosporin, sirolimus) because of increased blood concentrations of the immunosuppressants, and statins because of the elevated risk of dose-dependent AEs including rhabdomyolysis. Dose reductions may be required with other ASMs including phenobarbital, primidone, phenytoin, carbamazepine, diazepam, ethosuxamide and tiagabine. In addition, concomitant midazolam, triazolam or alprazolam can cause excessive sedation. Furthermore, a dose adjustment may be required with drugs metabolised by CYP2D6 including beta-blockers, 
antidepressants (e.g. fluoxetine, paroxetine, sertraline, imipramine, clomipramine), antipsychotics (haloperidol) and analgesics (codeine, dextromethorphan, tramadol).

\section{Highly Purified Cannabidiol (CBD)}

Highly purified CBD has received approval as an adjunctive therapy for seizures associated with DS, with LGS and most recently, for tuberous sclerosis complex (TSC). It was first approved in the USA in 2018 (Epidiolex ${ }^{\circledR}$ ), and in the EU in 2019 (Epidyolex $\left.{ }^{\circledR}\right)$; in the EU, it is indicated in conjunction with CLB (except for TSC where it is approved without the combination of CLB) (Table 1) [60, 61]. In the USA, it is approved for use in patients aged 1 year and older, while in the EU it is approved for patients aged 2 years and older. As of 2021, Epidiolex/Epidyolex ${ }^{\circledR}$ is the only CBD formulation that has been approved by the US Food and Drug Administration and the European Medicines Agency (referred to as CBD throughout). It is a highly purified, plant-derived, CBD oil-based solution that does not contain tetrahydrocannabinol, and as such does not have euphoric or intrusive side effects. Cannabidiol has mechanisms of action that are distinct from other ASMs (Fig. 4): it modulates intracellular calcium via both G-protein-coupled receptor 55 and transient receptor potential vanilloid 1, and also inhibits adenosine cellular uptake via the equilibrative nucleoside transporter 1 [62].

Cannabidiol is available as an oral solution at $100 \mathrm{mg} /$ $\mathrm{mL}$. It is given twice a day, with a recommended starting dose of $5 \mathrm{mg} / \mathrm{kg} /$ day for 1 week, followed by an increase to a maintenance dose of $10 \mathrm{mg} / \mathrm{kg} / \mathrm{day}$. Thereafter, it can be gradually titrated by $5 \mathrm{mg} / \mathrm{kg} /$ day up to a maximum of $20 \mathrm{mg} / \mathrm{kg} /$ day depending on clinical response and tolerability. It has been noted that slow titration of CBD, i.e. reaching a target dose of $10 \mathrm{mg} / \mathrm{kg} /$ day within at least 1 month and then gradually increasing to a maximum dose of $20 \mathrm{mg} / \mathrm{kg} /$ day, results in better tolerance with equivalent efficacy [63]. As food may increase the concentrations of CBD, it should consistently be taken either with or without food, with a preference for taking it with food at approximately the same time each day [64].

The efficacy and safety of CBD in DS was evaluated in two phase III, double-blind, placebo controlled RCTs (GWPCARE1 [CBD $20 \mathrm{mg} / \mathrm{kg} /$ day] and GWPCARE2 [CBD 10 and $20 \mathrm{mg} / \mathrm{kg} / \mathrm{day}]$ ) [65, 66]. In a highly refractory population (a median of four prior ASMs and three concomitant ASMs), responder rates were significantly higher compared with placebo (42.6-49.3\% vs $26.2-27.1 \%$ ), and were even higher in a subgroup of patients taking concomitant CLB (47.5-62.5\% vs $23.7-36.6 \%)$. Because of this potential enhanced efficacy with CLB, the approval from the European Medicines Agency stipulates that CBD be used in conjunction with CLB. Cannabidiol was also associated with improvements in overall condition assessed using the Caregiver Global Impression of Change, as well as increases in seizure-free days. Longer term efficacy and safety (up to 3 years) has been determined in an open-label extension of the pivotal phase III trials $[67,68]$, and importantly from an expanded access programme confirming its efficacy in realworld clinical practice [69-72].

Cannabidiol has been generally well tolerated across studies in patients with DS and LGS; the most common AEs from a pooled analysis were somnolence (particularly with concomitant CLB, see below), decreased appetite, diarrhoea, fatigue, malaise, asthenia, rash, insomnia and infections [60, 61]. Of note, a low-fibre diet may be considered during uptitration if the patient experiences diarrhoea.

Bi-directional interactions occur with CLB producing an increase in plasma concentrations of 7-OH-CBD (the major metabolite of CBD) and of nCLB by three-fold, a reason behind its enhanced efficacy compared with $\mathrm{CBD}$ without CLB, although it should be noted that CBD without CLB is also efficacious (Table 3) [73-75]. However, the interaction may also lead to an increase in AEs, particularly somnolence and sedation, requiring a dose reduction of CLB. In addition, CBD is associated with elevations of liver transaminases particularly in patients taking concomitant VPA; patients taking higher doses of CBD, and to a lesser extent concomitant CLB also had an increased incidence of transaminase elevations [60, 61]. Because of a risk of liver injury, serum transaminases (alanine aminotransferase and aspartate transaminase) and total bilirubin levels should be obtained prior to initiating CBD, after 1 month, 3 months and 6 months and then periodically or as clinically required (Table 2) [60, 61]. More frequent monitoring may be required in patients taking concomitant VPA or in those with elevated liver enzymes at baseline. Cases of thrombocytopenia have also been observed in some patients taking concomitant CBD and VPA [76]. With regard to other drug-drug interactions, a dose increase of CBD may be required if co-administered with medications that are strong inducers of CYP3A4 (e.g. enzalutamide, phenytoin) or CYP2C19 (e.g. rifampin, carbamazepine, phenobarbital, phenytoin, St. John's wort) [60, 61, 77].

\section{Fenfluramine (FFA)}

Fenfluramine as an add-on therapy was approved for the treatment of seizures associated with DS in mid-2020 in the USA and in late 2020 in the EU [78, 79]. Fenfluramine is a repurposed drug, having originally been used in adults at high doses (up to $120 \mathrm{mg} /$ day) in combination with phentermine (fen-phen) as a therapy for obesity until 1997 when it was withdrawn because of increased cases of cardiac valve 
disease, although it should be noted that phentermine also has an impact on valvular disease [80]. However, because of its role in stimulating the central serotoninergic pathways, as well as a few early case reports indicating its efficacy in patients with seizure disorders, FFA continued to be investigated as an ASM, with studies proceeding in Belgium where a Royal Decree was issued to allow FFA to be used at low doses in children with intractable epilepsy [80]. These studies, with favourable results in patients with DS [81, 82], were instrumental in paving the way for further clinical development that ultimately led to regulatory approval in patients with DS.

Fenfluramine is available as a $2.2-\mathrm{mg} / \mathrm{mL}$ oral solution. It is taken twice daily, with different dosing schedules according to whether the patient is taking STP because of an interaction whereby STP increases plasma concentrations of FFA and decreases its metabolite, norfenfluramine (Table 3) [83]. In both instances (i.e. with or without STP), an initial dose of $0.2 \mathrm{mg} / \mathrm{kg} /$ day is recommended, increased to $0.4 \mathrm{mg} / \mathrm{kg} /$ day after 7 days; $0.4 \mathrm{mg} / \mathrm{kg} /$ day is the recommended maintenance dose in patients taking STP, whereas in patients not taking STP, a further increase to the suggested maintenance dose of $0.7 \mathrm{mg} / \mathrm{kg} / \mathrm{day}$ can occur after another 7 days [78]. Patients should not exceed the maximum daily dose of $17 \mathrm{mg}$ when taking STP or $26 \mathrm{mg}$ when not taking STP.

Fenfluramine has a unique mechanism of action compared with other ASMs. Fenfluramine targets the serotonergic system, in multiple ways including promoting serotonin $(5-\mathrm{HT})$ release into the synapse, inhibiting the serotonin transporter, and acting as an agonist at multiple 5-HT receptors [84]. In addition, FFA was found to be a positive modulator of the sigma-1 receptor, a molecular chaperone that has roles in neuronal signalling; studies in a mouse model suggested that this mechanism of action could have implications for improved cognitive functions of spatial and contextual learning [85].

The efficacy and safety of FFA have been evaluated in three pivotal, phase III, double-blind, placebo-controlled RCTs in patients aged 2-18 years with DS; two trials conducted in patients without STP were identical and the results were merged [86], while the other trial exclusively included patients taking STP [87]. In patients who were highly drug resistant (baseline convulsive seizure frequencies of 14-20 per month despite the majority taking three or more concomitant ASMs), $\geq 50 \%$ responder rates for convulsive seizures were significantly higher compared with placebo (68-38\% vs 5-12\%). Fenfluramine was also associated with significantly longer seizure-free intervals, a significantly higher number of convulsive seizure-free days and improvements in achieving near-seizure freedom $(<1$ seizure over the 14-week treatment period) [86-88]. In addition, compared with placebo, more patients were rated as "very much" or "much improved" according to the Clinical
Global Impression-Improvement. Data from an open-label extension trial have shown that reductions in convulsive seizure frequency are sustained over a median duration of treatment of 3 years [89]. Furthermore, real-world data obtained via a compassionate use programme have shown that FFA had a good retention rate, and as well as reductions in seizures and seizure days per months, FFA was associated with reductions in episodes of SE and reductions in the number of concomitant ASMs [90, 91]. Moreover, case reports have described the use of FFA for the successful treatment of nonconvulsive SE in an 8-year-old patient [92] and superrefractory SE in a child [93] and a 20-year-old adult [94]. Additionally, FFA may be associated with reductions in allcause and sudden unexpected death in epilepsy mortality rates in patients with DS [95].

Fenfluramine is generally well tolerated. In-line with the anorexigenic effects of FFA that were harnessed when it was used as an anti-obesity therapy, decreased appetite and weight loss are among the most common AEs [78, 79]. Decreased weight is dose dependent, and an additive effect has been observed when FFA is combined with other ASMs such as STP or TPM. However, most patients resume weight gain over time while continuing treatment. Other common AEs include diarrhoea, fatigue, lethargy, pyrexia and upper respiratory tract infection.

Importantly, no signs of heart valve disease have been documented to date [96]. However, echocardiogram monitoring should be conducted before initiating FFA and then every 6 months for the first 2 years, with annual echocardiograms thereafter $[78,79]$. In addition, FFA is contraindicated in patients with aortic or mitral valvular heart disease and pulmonary arterial hypertension. In the EU, clinicians will need to register with a controlled access programme before initiating FFA for the first time [79]. The controlled access programme is a requirement of the European Medicines Agency to ensure that clinicians do not use FFA offlabel in weight management in obese patients and to confirm the need for periodic cardiac monitoring. Similarly, in the USA, clinicians, pharmacies and patients need to enrol in the REMS (Risk Evaluation and Mitigation Strategy) programme, which provides education about the risks of valvular heart disease and pulmonary arterial hypertension and the need for monitoring [78]. Other warnings include serotonin syndrome, a potentially fatal condition caused by excessive accumulation of serotonin in the body, which can occur particularly if co-administered with other serotonergic drugs (Table 2) [78, 79]. No particular requirements for dose adjustments of the ASMs of relevance for DS have been documented (Table 3), except for observations that bromide concentrations may be increased in some patients treated with FFA + bromide (expert opinion). Of note, data from the compassionate use programme in Europe suggest that FFA use can ultimately lead to reductions in the ASM drug 
load, associated with reductions in concomitant ASM use or in dose reductions [90, 91]. Finally, an increase in FFA dosage is recommended when co-administered with rifampin or a strong CYP1A2 and CYP2B6 inducer. Similar to CBD combinations with VPA, FFA may exacerbate VPA-induced thrombocytopenia [97].

\section{Topiramate (TPM)}

Topiramate, an ASM with a broad range of antiseizure activity, is indicated as adjunctive therapy in persons aged 2 years and above with partial-onset seizures with or without secondary generalisation or primary generalised tonicclonic seizures and for the treatment of seizures associated with LGS [98, 99]. Its mechanisms of action relating to seizure control are complex and not fully elucidated, but they may include blocking voltage-dependent sodium channels, enhancing the GABAergic pathway, antagonizing the glutamate receptor (the AMPA/kainate subtype), and inhibiting the carbonic anhydrase enzymes II and IV (Fig. 4) [100, 101].

Topiramate is available as capsules, film-coated tablets, an oral solution and sprinkle capsules; the latter can be given with (sprinkled over) a small amount of soft food. Topiramate is administered twice daily starting at $1-2 \mathrm{mg} /$ $\mathrm{kg} /$ day, with doses gradually increased to a target dosage of $5-10 \mathrm{mg} / \mathrm{kg} /$ day depending on tolerance and efficacy. Slow titration is important to reduce AEs.

Evidence of efficacy in patients with DS is derived from two small, prospective, open-label studies and a handful of small retrospective observational studies that have reported $>50 \%$ responder rates of between $35 \%$ and $78 \%$ [14, 102]. Larger RCTs have been conducted in patients with LGS and in patients with partial-onset or primary generalised tonicclonic seizures [98, 99, 103].

Topiramate has a generally acceptable tolerability and safety profile. A pooled analysis of TPM as an adjunct therapy across the epilepsy/LGS trials showed that the most common AEs were somnolence, fatigue, nausea, dizziness, ataxia, anorexia, paresthesia, anorexia, weight loss, and various cognitive/neuropsychiatric problems including nervousness, psychomotor slowing, difficulty with memory, speech disorders/related speech problems and personality disorder (behavioural problems) [98, 99]. Tolerability may be improved by slow titration with 2-week intervals [104]. Topiramate is also associated with a number of warnings requiring monitoring including visual defects, oligohidrosis and hyperthermia, metabolic acidosis, hyperammonaemia/ encephalopathy (especially with concomitant VPA) and kidney stones (Table 2) [98, 99]. Regarding the latter, staying hydrated by drinking plenty of fluids minimises the risk of kidney stones. The addition of TPM does not need any particular adaptation of dosages for the other ASMs used in DS (Table 3) [98, 99]. Decreased efficacy of oestrogencontaining contraceptives has been reported with high doses of TPM ( $\geq 200 \mathrm{mg}$ ), and in those situations non-hormonal forms of contraception are recommended (Table S1 of the ESM).

\section{Bromide}

Bromide is one of the oldest ASMs dating back to 1857; it lost favour for the treatment of seizures after the discovery of phenobarbital in 1912, but had a resurgence in some countries following reports in the 1980s and 1990s of efficacy in refractory epileptic syndromes. Bromide is not available in many countries; however, in Germany and Japan, it is approved for patients with severe epilepsies of infancy and early childhood presenting with generalised tonic-clonic seizures, including DS [105]. Its mechanisms of action are not fully understood; however, it may increase GABA-ergic inhibition [105].

The target dosage of bromide is between 30 and $70 \mathrm{mg} /$ $\mathrm{kg} /$ day [depending on age (Table 2)], given orally generally in two equal doses. It is available predominantly as potassium bromide tablets. The evidence base related to the efficacy of bromide in DS comes from a number of retrospective studies, which have reported $>50 \%$ responder rates of between $28 \%$ and $78 \%$ [30-32, 106, 107], together with its common use in some countries, thus inferring good efficacy [15].

Bromide is generally well tolerated at the low doses currently prescribed in children with refractory epilepsy. Adverse events can typically include loss of appetite, weight loss, ataxia, tremor, sedation, tiredness, cognitive slowing, disorientation, stupor, respiratory tract infections, gastritis and gastric ulcers. The risk of developing gastric ulcers can be reduced by using gastric acid-resistant capsules that can be prepared by the pharmacist using the tablets or potassium bromide salt. In addition, skin disturbances can occur, which may require the dosage to be reduced if troublesome, including rash, acne, ulcerations and bromoderma tuberosum (vegetating inflammation). The latter is rare but can be serious leading to scars, and because it does not appear to be dose dependent, it requires cessation of bromide treatment.

Bromide does not undergo hepatic metabolism and it is not subject to the same number of drug-drug interactions as other ASMs [105, 108]. However, a drug-food interaction can occur with the chloride ion, especially with table salt (sodium chloride), and as such it is recommended that a consistent and limited amount of sodium chloride be ingested. It is also important that prior to initiating bromide, renal function and electrolyte disturbances should be assessed and regular examinations are required to determine serum bromide 
concentrations. Furthermore, electrolyte imbalances during treatment (e.g. due to fluid loss because of severe vomiting, diarrhoea or excessive sweating) can also lead to increased AEs, requiring careful monitoring. In the case of an infection, children might require only half of their prescribed dose to prevent side effects caused by the accumulation of bromide in the body. Clinicians should also be aware that chloride levels may be falsely elevated in blood tests due to cross-reactivity with bromide during the testing procedure.

\section{Other Anti-Seizure Medications (ASMs) and Clinical Trials}

Other ASMs that could be considered include levetiracetam, brivaracetam, perampanel, zonisamide and ethosuximide; however, it should be noted that there is little evidence of their efficacy for controlling seizures in DS. Of note, ethosuximide is generally the preferred treatment for patients with absence seizures, with studies finding it more effective and/or more tolerable than VPA and lamotrigine in children with newly diagnosed childhood absence epilepsy [109, 110]. Furthermore, studies reporting on prescription patterns show that levetiracetam is frequently discontinued in patients with DS over time [111], suggesting a lack of longterm efficacy and/or tolerability, while caregivers of patients with DS scored it as only "somewhat effective" [112]. Practical details of these ASMs are provided in Table S2 of the ESM. Of particular importance in DS is that some ASMs tend to exacerbate seizures in patients with DS and should generally be avoided; these include sodium channel blockers such as carbamazepine, eslicarbazepine acetate, lacosamide and lamotrigine, oxcarbazepine and phenytoin, and ASMs acting on GABA, such as tiagabine, vigabatrin, gabapentin and pregabalin (Fig. 2).

Enrolling patients in clinical trials is also a consideration. Of the investigational agents, soticlestat (OV935/TAK-935), a first-in-class suppressor of cholesterol 24-hydroxylase $(\mathrm{CH} 24 \mathrm{H})$, is furthest along in clinical development, with a phase III study planned in patients with DS and LGS following positive results from the phase II ELEKTRA trial (NCT03650452) and the ENDYMION trial [113]. Furthermore, technological advances in developing genetic therapies are being harnessed for the treatment of DS, including antisense oligonucleotides, CRISPR/Cas9-mediated genome editing, and gene regulation therapy that uses an adenoassociated virus vector containing a transcription factor to increase protein production [114]. Genetic therapies for DS aim to restore the expression of $\mathrm{Na}_{\mathrm{V}} 1.1$, and by addressing the underlying aetiology/pathophysiology they have the potential to dramatically slow or even stop disease progression across all or many aspects of the syndrome (i.e. the different types of seizures and cognitive dysfunction). Of note,
STK-001, an ASO designed to upregulate $\mathrm{Na}_{\mathrm{v}} 1.1$ from the functioning wild-type allele, has entered phase I/II clinical development, with other genetic therapies not far behind.

\section{Conclusions}

While we have provided an overview of the practical issues surrounding the use of ASMs for the treatment of seizures in patients with DS, there remain several unanswered questions for clinicians that require further evidence. First, there are currently no head-to-head trials of CBD vs FFA, and therefore the decision as to which option to choose is mainly based on clinicians' experience, individual patient factors and patient/caregiver preferences, as well as availability within individual countries. The latter is important to point out-not all treatments described here are available in all countries. Additionally, it is noteworthy that it is currently difficult to choose an ASM based on the seizure type, although ethosuximide is better suited for absence seizures. Second, evidence around improvements in cognitive dysfunction and in quality of life is still generally lacking, although there are the beginnings of evidence that FFA and CBD have the potential for improvements in these highly relevant treatment goals [115-117]. In addition, while it is known that the burden of illness and healthcare costs of DS are high [118-123], there is a lack of published evaluations regarding the cost effectiveness of the ASMs used to treat patients with DS. Furthermore, in clinical practice, ASMs may be given off-label to patients who are younger than the indicated age, and there is a lack of information about the dose, safety and tolerability in these cases. Finally, while the approval of STP, CBD and FFA undoubtedly represent tremendous progress, DS still poses a heavy burden for patients and their families. To make further progress, a number of challenges still need to be overcome including improving early diagnosis to allow for prompt and appropriate treatment, reductions in polypharmacy, improvements in quality of life, the availability of additional treatments for those who do not benefit on those currently available and the identification of biomarkers to aid the tailoring of treatments to the individual patient.

Supplementary Information The online version contains supplementary material available at https://doi.org/10.1007/s40263-022-00898-1.

Acknowledgements Medical writing assistance to identify and synthesise the data and to draft the first draft, under the direction of the authors, was provided by Amanda Prowse (Lochside Medical Communications Ltd). Graphics support was provided by Emma Melchor (Emma Melchor Illustration). 


\section{Declarations}

Funding Open Access funding enabled and organized by Projekt DEAL. Medical writing assistance was funded by GW Pharma (Germany) $\mathrm{GmbH}$.

Conflicts of interest/competing interests A. Strzelczyk reports personal fees and grants from Angelini Pharma/Arvelle Therapeutics, Desitin Arzneimittel, Eisai, GW Pharmaceuticals, Marinus Pharmaceuticals, UCB Pharma, UNEEG medical, and Zogenix. S. SchubertBast reports personal fees from Eisai, Desitin Pharma, GW Pharmaceuticals, LivaNova, UCB Pharma, and Zogenix.

Ethics approval Not applicable.

Consent to participate Not applicable.

Consent for publication Not applicable.

Availability of data and material Not applicable.

Code availability Not applicable.

Authors' Contributions Both authors reviewed the literature, drafted the manuscript, generated the figures, and assume full responsibility for the final publication.

Open Access This article is licensed under a Creative Commons Attribution-NonCommercial 4.0 International License, which permits any non-commercial use, sharing, adaptation, distribution and reproduction in any medium or format, as long as you give appropriate credit to the original author(s) and the source, provide a link to the Creative Commons licence, and indicate if changes were made. The images or other third party material in this article are included in the article's Creative Commons licence, unless indicated otherwise in a credit line to the material. If material is not included in the article's Creative Commons licence and your intended use is not permitted by statutory regulation or exceeds the permitted use, you will need to obtain permission directly from the copyright holder. To view a copy of this licence, visit http://creativecommons.org/licenses/by-nc/4.0/.

\section{References}

1. Strzelczyk A, Schubert-Bast S. Therapeutic advances in Dravet syndrome: a targeted literature review. Expert Rev Neurother. 2020;20(10):1065-79.

2. Li W, Schneider AL, Scheffer IE. Defining Dravet syndrome: an essential pre-requisite for precision medicine trials. Epilepsia. 2021;62(9):2205-17.

3. Dravet C. The core Dravet syndrome phenotype. Epilepsia. 2011;52(Suppl. 2):3-9.

4. Catterall WA, Kalume F, Oakley JC. NaV1.1 channels and epilepsy. J Physiol. 2010;588(Pt 11):1849-59.

5. Selvarajah A, Zulfiqar-Ali Q, Marques P, Rong M, Andrade DM. A systematic review of adults with Dravet syndrome. Seizure. 2021;87:39-45.

6. Licheni SH, McMahon JM, Schneider AL, Davey MJ, Scheffer IE. Sleep problems in Dravet syndrome: a modifiable comorbidity. Dev Med Child Neurol. 2018;60(2):192-8.

7. Shmuely S, Sisodiya SM, Gunning WB, Sander JW, Thijs RD. Mortality in Dravet syndrome: a review. Epilepsy Behav. 2016;64(Pt A):69-74.
8. Nabbout R, Chemaly N, Chipaux M, Barcia G, Bouis C, Dubouch $\mathrm{C}$, et al. Encephalopathy in children with Dravet syndrome is not a pure consequence of epilepsy. Orphanet J Rare Dis. 2013;13(8): 176 .

9. Brunklaus A, Ellis R, Reavey E, Forbes GH, Zuberi SM. Prognostic, clinical and demographic features in SCN1A mutationpositive Dravet syndrome. Brain. 2012;135(Pt 8):2329-36.

10. Lagae L, Brambilla I, Mingorance A, Gibson E, Battersby A. Quality of life and comorbidities associated with Dravet syndrome severity: a multinational cohort survey. Dev Med Child Neurol. 2018;60(1):63-72.

11. Ragona F, Brazzo D, De Giorgi I, Morbi M, Freri E, Teutonico F, et al. Dravet syndrome: early clinical manifestations and cognitive outcome in 37 Italian patients. Brain Dev. 2010;32(1):71-7.

12. Wolff M, Cassé-Perrot C, Dravet C. Severe myoclonic epilepsy of infants (Dravet syndrome): natural history and neuropsychological findings. Epilepsia. 2006;47(Suppl. 2):45-8.

13. Strzelczyk A, Frey K, Rosenow F, Schubert-Bast S. Anticonvulsant Agents: Cannabidiol and Fenfluramine. In: Riederer P, Laux G, Mulsant B, Le W, Nagatsu T, editors. NeuroPsychopharmacotherapy. Cham: Springer International Publishing; 2022. p. 1-15.

14. Wirrell EC, Nabbout R. Recent advances in the drug treatment of Dravet syndrome. CNS Drugs. 2019;33(9):867-81.

15. Schubert-Bast $S$, Wolff M, Wiemer-Kruel A, von Spiczak S, Trollmann R, Reif PS, et al. Seizure management and prescription patterns of anticonvulsants in Dravet syndrome: a multicenter cohort study from Germany and review of literature. Epilepsy Behav. 2019;98(Pt A):88-95.

16. Lagae L. Dravet syndrome. Curr Opin Neurol. 2021;34(2):213-8.

17. Schoonjans AS, Ceulemans B. Dravet syndrome: toward an optimal and disease-specific treatment. Z Epileptol. 2021;34(2):146-53.

18. Brigo F, Striano P, Balagura G, Belcastro V. Emerging drugs for the treatment of Dravet syndrome. Expert Opin Emerg Drugs. 2018;23(4):261-9.

19. Fallah A. Moving beyond evidence-based medicine: incorporating patient values and preferences. Epilepsy Behav. 2015;53:209-10.

20. Kalski M, Schubert-Bast S, Kieslich M, Leyer AC, Polster T, Herting A, et al. Klinische Charakteristika, Ressourcenverbrauch, Lebensqualität und Versorgungssituation beim Dravet-Syndrom in Deutschland. Z Epileptol. 2019;32(4):326-38.

21. Wheless JW, Fulton SP, Mudigoudar BD. Dravet syndrome: a review of current management. Pediatr Neurol. 2020;107:28-40.

22. Nalivaeva NN, Belyaev ND, Turner AJ. Sodium valproate: an old drug with new roles. Trends Pharmacol Sci. 2009;30(10):509-14.

23. Marson A, Burnside G, Appleton R, Smith D, Leach JP, Sills G, et al. The SANAD II study of the effectiveness and cost-effectiveness of valproate versus levetiracetam for newly diagnosed generalised and unclassifiable epilepsy: an open-label, non-inferiority, multicentre, phase 4 , randomised controlled trial. Lancet. 2021;397(10282):1375-86

24. Marson AG, Al-Kharusi AM, Alwaidh M, Appleton R, Baker GA, Chadwick DW, et al. The SANAD study of effectiveness of valproate, lamotrigine, or topiramate for generalised and unclassifiable epilepsy: an unblinded randomised controlled trial. Lancet. 2007;369(9566):1016-26.

25. Cook M, Murphy M, Bulluss K, D'Souza W, Plummer C, Priest E, et al. Anti-seizure therapy with a long-term, implanted intracerebroventricular delivery system for drug-resistant epilepsy: a first-in-man study. EClinicalMedicine. 2020;22:100326.

26. Depakene (valproic acid): prescribing information. Available from: https://www.accessdata.fda.gov/drugsatfda_docs/label/ 2016/018081s065_018082s0481bl.pdf. Accessed 14 Jan 2021. 
27. Epilim (sodium valproate): summary of product characteristics. Available from: https://www.medicines.org.uk/emc/product/ 1446/smpc\#gref. Accessed Jun 2021.

28. Bolszak M, Anttonen AK, Komulainen T, Hinttala R, Pakanen $\mathrm{S}$, Sormunen $\mathrm{R}$, et al. Digenic mutations in severe myoclonic epilepsy of infancy. Epilepsy Res. 2009;85(2-3):300-4.

29. Craig AK, de Menezes MS, Saneto RP. Dravet syndrome: patients with co-morbid SCN1A gene mutations and mitochondrial electron transport chain defects. Seizure. 2012;21(1):17-20.

30. Dressler A, Trimmel-Schwahofer P, Reithofer E, Muhlebner A, Groppel G, Reiter-Fink E, et al. Efficacy and tolerability of the ketogenic diet in Dravet syndrome: comparison with various standard antiepileptic drug regimen. Epilepsy Res. 2015;109:81-9.

31. Inoue Y, Ohtsuka Y, Oguni H, Tohyama J, Baba H, Fukushima $\mathrm{K}$, et al. Stiripentol open study in Japanese patients with Dravet syndrome. Epilepsia. 2009;50(11):2362-8.

32. Shi XY, Tomonoh Y, Wang WZ, Ishii A, Higurashi N, Kurahashi $\mathrm{H}$, et al. Efficacy of antiepileptic drugs for the treatment of Dravet syndrome with different genotypes. Brain Dev. 2016;38(1):40-6.

33. Frisium (clobazam): summary of product characteristics. https:// www.medicines.org.uk/emc/product/1574/smpc\#gref. Accessed Jun 2021.

34. Onfi (clobazam): prescribing information. https://www.acces sdata.fda.gov/drugsatfda_docs/label/2016/203993s005lbl.pdf. Accessed Jun 2021.

35. Gauthier AC, Mattson RH. Clobazam: a safe, efficacious, and newly rediscovered therapeutic for epilepsy. CNS Neurosci Ther. 2015;21(7):543-8.

36. Sympazan (clobazam oral film). https://www.accessdata.fda. gov/drugsatfda_docs/label/2018/210833s000lbl.pdf. Accessed Jun 2021.

37. Ng YT, Conry JA, Drummond R, Stolle J, Weinberg MA. Randomized, phase III study results of clobazam in Lennox-Gastaut syndrome. Neurology. 2011;77(15):1473-81.

38. Conry JA, Ng YT, Kernitsky L, Mitchell WG, Veidemanis R, Drummond R, et al. Stable dosages of clobazam for LennoxGastaut syndrome are associated with sustained drop-seizure and total-seizure improvements over 3 years. Epilepsia. 2014;55(4):558-67.

39. Nickels KC, Wirrell EC. Stiripentol in the management of epilepsy. CNS Drugs. 2017;31(5):405-16.

40. Fisher JL. Interactions between modulators of the GABA(A) receptor: stiripentol and benzodiazepines. Eur J Pharmacol. 2011;654(2):160-5.

41. Giraud C, Treluyer JM, Rey E, Chiron C, Vincent J, Pons G, et al. In vitro and in vivo inhibitory effect of stiripentol on clobazam metabolism. Drug Metab Dispos. 2006;34(4):608-11.

42. Wirrell EC, Laux L, Franz DN, Sullivan J, Saneto RP, Morse RP, et al. Stiripentol in Dravet syndrome: results of a retrospective U.S. study. Epilepsia. 2013;54(9):1595-604.

43. May TW, Boor R, Mayer T, Jürgens U, Rambeck B, Holert N, et al. Concentrations of stiripentol in children and adults with epilepsy: the influence of dose, age, and comedication. Ther Drug Monit. 2012;34(4):390-7.

44. Diacomit: prescribing information. https://www.accessdata.fda. gov/drugsatfda_docs/label/2018/206709s000,207223s000lbl.pdf. Accessed Mar 2020.

45. Diacomit: summary of product characteristics. https://www. medicines.org.uk/emc/product/10300/smpc. Accessed Apr 2020.

46. Brigo F, Igwe SC, Bragazzi NL. Stiripentol add-on therapy for drug-resistant focal epilepsy. Cochrane Database Syst Rev. 2020;5(5):CD009887.

47. Chiron C, Marchand MC, Tran A, Rey E, d'Athis P, Vincent $\mathrm{J}$, et al. Stiripentol in severe myoclonic epilepsy in infancy: a randomised placebo-controlled syndrome-dedicated trial. STICLO Study Group. Lancet. 2000;356(9242):1638-42.

48. European Medicines Agency. Diacomit: EPAR - scientific discussion. https://www.ema.europa.eu/en/documents/scientific-discu ssion/diacomit-epar-scientific-discussion_en.pdf. Accessed Mar 2020.

49. Thanh TN, Chiron C, Dellatolas G, Rey E, Pons G, Vincent J, et al. Long-term efficacy and tolerance of stiripentaol in severe myoclonic epilepsy of infancy (Dravet's syndrome). Arch Pediatr. 2002;9(11):1120-7.

50. Chiron C, Helias M, Kaminska A, Laroche C, de Toffol B, Dulac O, et al. Do children with Dravet syndrome continue to benefit from stiripentol for long through adulthood? Epilepsia. 2018;59(9):1705-17.

51. Inoue Y, Ohtsuka Y. Effectiveness of add-on stiripentol to clobazam and valproate in Japanese patients with Dravet syndrome: additional supportive evidence. Epilepsy Res. 2014;108(4):725-31.

52. Inoue Y, Ohtsuka Y. Long-term safety and efficacy of stiripentol for the treatment of Dravet syndrome: a multicenter, open-label study in Japan. Epilepsy Res. 2015;113:90-7.

53. Yamada M, Suzuki K, Matsui D, Inoue Y, Ohtsuka Y. Long-term safety and effectiveness of stiripentol in patients with Dravet syndrome: interim report of a post-marketing surveillance study in Japan. Epilepsy Res. 2021;170:106535.

54. Habermehl L, Mross PM, Krause K, Immisch I, Chiru D, Zahnert $\mathrm{F}$, et al. Stiripentol in the treatment of adults with focal epilepsya retrospective analysis. Seizure. 2021;88:7-11.

55. Frampton JE. Stiripentol: a review in Dravet syndrome. Drugs. 2019;79(16):1785-96.

56. Chiron C. Stiripentol for the treatment of seizures associated with Dravet syndrome. Expert Rev Neurother. 2019;19(4):301-10.

57. Balestrini S, Sisodiya SM. Audit of use of stiripentol in adults with Dravet syndrome. Acta Neurol Scand. 2017;135(1):73-9.

58. De Liso P, Chemaly N, Laschet J, Barnerias C, Hully M, Leunen D, et al. Patients with Dravet syndrome in the era of stiripentol: a French cohort cross-sectional study. Epilepsy Res. 2016;125:42-6.

59. Zulfiqar Ali Q, Marques P, Selvarajah A, Tabarestani S, Sadoway T, Andrade DM. Starting stiripentol in adults with Dravet syndrome? Watch for ammonia and carnitine. Epilepsia. 2020;61(11):2435-41.

60. Epidiolex: prescribing information. https://www.accessdata.fda. gov/drugsatfda_docs/label/2020/210365s005s006s007lbl.pdf. Accessed Jun 2021.

61. Epidyolex: summary of product characteristics. https://www. medicines.org.uk/emc/product/10781\#gref. Accessed Jun 2021.

62. Gray RA, Whalley BJ. The proposed mechanisms of action of CBD in epilepsy. Epilept Disord. 2020;22(S1):10-5.

63. D'Onofrio G, Kuchenbuch M, Hachon-Le Camus C, Desnous B, Staath V, Napuri S, et al. Slow titration of cannabidiol add-on in drug-resistant epilepsies can improve safety with maintained efficacy in an open-label study. Front Neurol. 2020;11:829.

64. Abu-Sawwa R, Stehling C. Epidiolex (cannabidiol) primer: frequently asked questions for patients and caregivers. J Pediatr Pharmacol Ther. 2020;25(1):75-7.

65. Devinsky O, Cross JH, Laux L, Marsh E, Miller I, Nabbout R, et al. Trial of cannabidiol for drug-resistant seizures in the Dravet syndrome. N Engl J Med. 2017;376(21):2011-20.

66. Miller I, Scheffer IE, Gunning B, Sanchez-Carpintero R, GilNagel A, Perry MS, et al. Dose-ranging effect of adjunctive oral cannabidiol vs placebo on convulsive seizure frequency in Dravet syndrome: a randomized clinical trial. JAMA Neurol. 2020;77(5):613-21.

67. Devinsky O, Nabbout R, Miller I, Laux L, Zolnowska M, Wright $\mathrm{S}$, et al. Long-term cannabidiol treatment in patients 
with Dravet syndrome: an open-label extension trial. Epilepsia. 2019;60(2):294-302.

68. Scheffer IE, Halford JJ, Miller I, Nabbout R, Sanchez-Carpintero $\mathrm{R}$, Shiloh-Malawsky Y, et al. Add-on cannabidiol in patients with Dravet syndrome: results of a long-term open-label extension trial. Epilepsia. 2021;62(10):2505-17.

69. Iannone LF, Arena G, Battaglia D, Bisulli F, Bonanni P, Boni A, et al. Results from an Italian expanded access program on cannabidiol treatment in highly refractory Dravet syndrome and Lennox-Gastaut syndrome. Front Neurol. 2021;12:673135.

70. Laux LC, Bebin EM, Checketts D, Chez M, Flamini R, Marsh $\mathrm{ED}$, et al. Long-term safety and efficacy of cannabidiol in children and adults with treatment resistant Lennox-Gastaut syndrome or Dravet syndrome: expanded access program results. Epilepsy Res. 2019;154:13-20.

71. Park YD, Linder DF, Pope J, Flamini JR, Moretz K, Diamond MP, et al. Long-term efficacy and safety of cannabidiol (CBD) in children with treatment-resistant epilepsy: results from a state-based expanded access program. Epilepsy Behav. 2020;112:107474.

72. Patel S, Grinspoon R, Fleming B, Skirvin LA, Wade C, Wolper $\mathrm{E}$, et al. The long-term efficacy of cannabidiol in the treatment of refractory epilepsy. Epilepsia. 2021;62(7):1594-603.

73. Bialer M, Perucca E. Does cannabidiol have antiseizure activity independent of its interactions with clobazam? An appraisal of the evidence from randomized controlled trials. Epilepsia. 2020;61(6):1082-9.

74. Gaston TE, Bebin EM, Cutter GR, Ampah SB, Liu Y, Grayson LP, et al. Drug-drug interactions with cannabidiol (CBD) appear to have no effect on treatment response in an open-label expanded access program. Epilepsy Behav. 2019;98(Pt A):201-6.

75. Lattanzi S, Trinka E, Striano P, Zaccara G, Del Giovane C, Nardone R, et al. Cannabidiol efficacy and clobazam status: a systematic review and meta-analysis. Epilepsia. 2020;61(6):1090-8.

76. McNamara NA, Dang LT, Sturza J, Ziobro JM, Fedak Romanowski EM, Smith GC, et al. Thrombocytopenia in pediatric patients on concurrent cannabidiol and valproic acid. Epilepsia. 2020;61(8):e85-9.

77. Brown JD, Winterstein AG. Potential adverse drug events and drug-drug interactions with medical and consumer cannabidiol (CBD) use. J Clin Med. 2019;8(7):989.

78. Fintepla: prescribing information. https://www.accessdata.fda. gov/drugsatfda_docs/label/2020/212102s000lbl.pdf. Accessed Jul 2020.

79. Fintepla: summary of product characteristics. https://www.medic ines.org.uk/emc/product/11998/smpc\#gref. Accessed Mar 2021.

80. Schoonjans AS, Ceulemans B. An old drug for a new indication: repurposing fenfluramine from an anorexigen to an antiepileptic drug. Clin Pharmacol Ther. 2019;106(5):929-32.

81. Ceulemans B, Boel M, Leyssens K, Van Rossem C, Neels P, Jorens PG, et al. Successful use of fenfluramine as an add-on treatment for Dravet syndrome. Epilepsia. 2012;53(7):1131-9.

82. Ceulemans B, Schoonjans AS, Marchau F, Paelinck BP, Lagae L. Five-year extended follow-up status of 10 patients with Dravet syndrome treated with fenfluramine. Epilepsia. 2016;57(7):e129-34.

83. Boyd B, Smith S, Gammaitoni A, Galer BS, Farfel GM. A phase I, randomized, open-label, single-dose, 3-period crossover study to evaluate the drug-drug interaction between ZX008 (fenfluramine $\mathrm{HCl}$ oral solution) and a regimen of stiripentol, clobazam, and valproate in healthy subjects. Int J Clin Pharmacol Ther. 2019;57(1):11-9.

84. Schoonjans A-S, Ceulemans B. A critical evaluation of fenfluramine hydrochloride for the treatment of Dravet syndrome. Expert Rev Neurother. 2021;1-14.
85. Martin P, de Witte PAM, Maurice T, Gammaitoni A, Farfel G, Galer B. Fenfluramine acts as a positive modulator of sigma-1 receptors. Epilepsy Behav. 2020;105:106989.

86. Lagae L, Sullivan J, Knupp K, Laux L, Polster T, Nikanorova M, et al. Fenfluramine hydrochloride for the treatment of seizures in Dravet syndrome: a randomised, double-blind, placebo-controlled trial. Lancet. 2020;394(10216):2243-54.

87. Nabbout R, Mistry A, Zuberi S, Villeneuve N, Gil-Nagel A, Sanchez-Carpintero R, et al. Fenfluramine for treatment-resistant seizures in patients with Dravet syndrome receiving stiripentolinclusive regimens: a randomized clinical trial. JAMA Neurol. 2019;77:300.

88. Sullivan J, Specchio N, Devinsky O, Auvin S, Perry MS, Strzelczyk A, et al. Fenfluramine significantly reduces day-to-day seizure burden by increasing number of seizure-free days and time between seizures in patients with Dravet syndrome: a timeto-event analysis. Epilepsia. 2022;63(1):130-8.

89. Scheffer IE, Devinsky O, Perry MS, Wheless JW, Thiele E, Wirrell E, et al. Efficacy and tolerability of adjunctive fintepla (fenfluramine hydrochloride) in an open-label extension study of dravet syndrome patients treated for up to 3 years. AES 2020 Virtual Meeting; 4-8 December, 2020.

90. Specchio N, Pietrafusa N, Doccini V, Trivisano M, Darra F, Ragona F, et al. Efficacy and safety of fenfluramine hydrochloride for the treatment of seizures in Dravet syndrome: a realworld study. Epilepsia. 2020;61(11):2405-14.

91. Strzelczyk A, Pringsheim M, Mayer T, Polster T, Klotz KA, Muhle H, et al. Efficacy, tolerability, and retention of fenfluramine for the treatment of seizures in patients with Dravet syndrome: compassionate use program in Germany. Epilepsia. 2021;62(10):2518-27.

92. Specchio N, Pietrafusa N, Ferretti A, Trivisano M, Vigevano F. Successful use of fenfluramine in nonconvulsive status epilepticus of Dravet syndrome. Epilepsia. 2020;61(4):831-3.

93. Trowbridge S, Poduri A, Olson H. Early diagnosis and experimental treatment with fenfluramine via the investigational new drug mechanism in a boy with Dravet syndrome and recurrent status epilepticus. Epileptic Disord. 2021;23(6):954-6.

94. Millett D, Pach S. Fenfluramine in the successful treatment of super-refractory status epilepticus in a patient with Dravet syndrome. Epilepsy Behav Rep. 2021;16:100461.

95. Cross JH, Galer BS, Gil-Nagel A, Devinsky O, Ceulemans B, Lagae L, et al. Impact of fenfluramine on the expected SUDEP mortality rates in patients with Dravet syndrome. Seizure. 2021;2(93):154-9.

96. Lai WW, Galer BS, Wong PC, Farfel G, Pringsheim M, Keane MG, et al. Cardiovascular safety of fenfluramine in the treatment of Dravet syndrome: analysis of an ongoing long-term open-label safety extension study. Epilepsia. 2020;61(11):2386-95.

97. Schoonjans A, Maes P, Ceulemans B. Aggravation of valproic acid induced thrombocytopenia after the introduction of fenfluramine, a case report. Seizure. 2021;93:60-2.

98. Topamax (topiramate): summary of product characteristics. https://www.medicines.org.uk/emc/product/1442/smpc\#gref. Accessed Jun 2021.

99. Topamax (topiramate): prescribing information. https://www. accessdata.fda.gov/drugsatfda_docs/label/2017/020505s057_ 020844s0481bl.pdf. Accessed Jun 2021.

100. Faught E. Topiramate in the treatment of partial and generalized epilepsy. Neuropsychiatr Dis Treat. 2007;3(6):811-21.

101. Michoulas A, Farrell K. Medical management of lennox-gastaut syndrome. CNS Drugs. 2010;24(5):363-74.

102. Nabbout R, Chemaly N, Chiron C, Kuchenbuch M. Safety considerations selecting antiseizure medications for the treatment of individuals with Dravet syndrome. Expert Opin Drug Saf. 2021;28:1-16. 
103. Sachdeo RC, Glauser TA, Ritter F, Reife R, Lim P, Pledger G. A double-blind, randomized trial of topiramate in Lennox-Gastaut syndrome. Neurology. 1999;52(9):1882-7.

104. Albsoul-Younes AM, Salem HA, Ajlouni SF, Al-Safi SA. Topiramate slow dose titration: improved efficacy and tolerability. Pediatr Neurol. 2004;31(5):349-52.

105. Bast T, Steinhoff BJ. Anticonvulsant Agents: Potassium Bromide. In: Riederer P, Laux G, Mulsant B, Le W, Nagatsu T, editors. NeuroPsychopharmacotherapy. Cham: Springer International Publishing; 2020. p. 1-7.

106. Lotte J, Haberlandt E, Neubauer B, Staudt M, Kluger GJ. Bromide in patients with SCN1A-mutations manifesting as Dravet syndrome. Neuropediatrics. 2012;43(1):17-21.

107. Ishii A, Watkins JC, Chen D, Hirose S, Hammer MF. Clinical implications of SCN1A missense and truncation variants in a large Japanese cohort with Dravet syndrome. Epilepsia. 2017;58(2):282-90.

108. Ryan M, Baumann RJ. Use and monitoring of bromides in epilepsy treatment. Pediatr Neurol. 1999;21(2):523-8.

109. Glauser TA, Cnaan A, Shinnar S, Hirtz DG, Dlugos D, Masur D, et al. Ethosuximide, valproic acid, and lamotrigine in childhood absence epilepsy: initial monotherapy outcomes at 12 months. Epilepsia. 2013;54(1):141-55.

110. Glauser TA, Cnaan A, Shinnar S, Hirtz DG, Dlugos D, Masur D, et al. Ethosuximide, valproic acid, and lamotrigine in childhood absence epilepsy. N Engl J Med. 2010;362(9):790-9.

111. Aras LM, Isla J, Mingorance-Le MA. The European patient with Dravet syndrome: results from a parent-reported survey on antiepileptic drug use in the European population with Dravet syndrome. Epilepsy Behav. 2015;44:104-9.

112. Villas N, Meskis MA, Goodliffe S. Dravet syndrome: characteristics, comorbidities, and caregiver concerns. Epilepsy Behav. 2017;74:81-6.

113. Takeda. Phase 2 ELEKTRA study of soticlestat (TAK-935/ OV935) meets primary endpoint reducing seizure frequency in children with Dravet syndrome or Lennox-Gastaut syndrome. 2020. http://www.takeda.com/newsroom/newsreleases/2020/ phase-2-elektra-study-of-soticlestat-tak-935ov935-meets-prima ry-endpoint-reducing-seizure-frequency-in-children-with-dravetsyndrome-or-lennox-gastaut-syndrome/. Accessed Aug 2020.

114. Higurashi N, Broccoli V, Hirose S. Genetics and gene therapy in Dravet syndrome. Epilepsy Behav. 2021;108043.

115. Amtmann D, Salem R, Gammaitoni A, Galer B, Wilkie D, Jensen M. Caregivers' perspectives on the long-term seizure- and non-seizure-related benefits of fenfluramine on patients with Dravet syndrome and their families (1529). Neurology. 2021;96(15 Suppl.):1529.

116. Metternich B, Wagner K, Geiger MJ, Hirsch M, Schulze-Bonhage A, Klotz KA. Cognitive and behavioral effects of cannabidiol in patients with treatment-resistant epilepsy. Epilepsy Behav. 2021;114(Pt A): 107558

117. Jensen MP, Salem R, Gammaitoni A, Galer B, Maruscak M, Wilkie $\mathrm{D}$, et al. The long-term effects of fenflramine on patients with Dravet syndrome and their families: a qualitative analysis. Acad Manage Care Pharm. 2021;2021.

118. Lagae L, Irwin J, Gibson E, Battersby A. Caregiver impact and health service use in high and low severity Dravet syndrome: a multinational cohort study. Seizure. 2019;65:72-9.

119. Strzelczyk A, Kalski M, Bast T, Wiemer-Kruel A, Bettendorf U, Kay L, et al. Burden-of-illness and cost-driving factors in Dravet syndrome patients and carers: a prospective, multicenter study from Germany. Eur J Paediatr Neurol. 2019;23(3):392-403.

120. Strzelczyk A, Schubert-Bast S, Bast T, Bettendorf U, Fiedler B, Hamer HM, et al. A multicenter, matched case-control analysis comparing burden-of-illness in Dravet syndrome to refractory epilepsy and seizure remission in patients and caregivers in Germany. Epilepsia. 2019;60(8):1697-710.

121. Strzelczyk A, Schubert-Bast S, Reese JP, Rosenow F, Stephani $\mathrm{U}$, Boor R. Evaluation of health-care utilization in patients with Dravet syndrome and on adjunctive treatment with stiripentol and clobazam. Epilepsy Behav. 2014;34:86-91.

122. Whittington MD, Knupp KG, Vanderveen G, Kim C, Gammaitoni A, Campbell JD. The direct and indirect costs of Dravet syndrome. Epilepsy Behav. 2018;80:109-13.

123. Schubert-Bast S, Kay L, Simon A, Wyatt G, Holland R, Rosenow F, Strzelczyk A. Epidemiology, healthcare resource use, and mortality in patients with probable Dravet syndrome: a population-based study on German health insurance data. Epilepsy Behav. 2022;126:108442.

124. Cross JH, Caraballo RH, Nabbout R, Vigevano F, Guerrini R, Lagae L. Dravet syndrome: treatment options and management of prolonged seizures. Epilepsia. 2019;60(Suppl. 3):S39-48. 